\title{
The quiet Sun magnetic field observed with ZIMPOL on THEMIS $\star$
}

\section{The probability density function}

\author{
V. Bommier ${ }^{1}$, M. Martínez González ${ }^{1, \star \star}$, M. Bianda ${ }^{2,3}$, H. Frisch $^{4}$, A. Asensio Ramos ${ }^{5}$, \\ B. Gelly ${ }^{6}$, and E. Landi Degl'Innocenti ${ }^{7}$
}

\author{
1 LERMA, Observatoire de Paris, ENS, UPMC, UCP, CNRS, Place Jules Janssen, 92190 Meudon, France \\ e-mail: V.Bommier@obspm.fr \\ 2 Istituto Ricerche Solari Locarno, via Patocchi, 6605 Locarno-Monti, Switzerland \\ Institute of Astronomy, ETH Zurich, 8092 Zurich, Switzerland \\ 4 Université de Nice, Observatoire de la Côte d'Azur, CNRS, Laboratoire Cassiopée, BP 4229, 06304 Nice Cedex 4, France \\ 5 Instituto de Astrofísica de Canarias, vía Láctea s/n, 38205 La Laguna, Tenerife, Spain \\ 6 Télescope Héliographique pour l'Étude du Magnétisme et des Instabilités Solaires, CNRS UPS 853 - THEMIS, vía Láctea s/n, \\ 38205 La Laguna, Tenerife, Spain \\ 7 Dipartimento di Astronomia e Scienza dello Spazio, Università degli Studi di Firenze, Largo E. Fermi 2, 50125 Firenze, Italy
}

Received 18 November 2008 / Accepted 16 July 2009

\begin{abstract}
Context. The quiet Sun magnetic field probability density function (PDF) remains poorly known. Modeling this field also introduces a magnetic filling factor that is also poorly known. With these two quantities, PDF and filling factor, the statistical description of the quiet Sun magnetic field is complex and needs to be clarified.

Aims. In the present paper, we propose a procedure that combines direct determinations and inversion results to derive the magnetic field vector and filling factor, and their PDFs.

Methods. We used spectro-polarimetric observations taken with the ZIMPOL polarimeter mounted on the THEMIS telescope. The target was a quiet region at disk center. We analyzed the data by means of the UNNOFIT inversion code, with which we inferred the distribution of the mean magnetic field $\alpha B, \alpha$ being the magnetic filling factor. The distribution of $\alpha$ was derived by an independent method, directly from the spectro-polarimetric data. The magnetic field PDF $p(B)$ could then be inferred. By introducing a joint PDF for the filling factor and the magnetic field strength, we have clarified the definition of the PDF of the quiet Sun magnetic field when the latter is assumed not to be volume-filling.

Results. The most frequent local average magnetic field strength is found to be $13 \mathrm{G}$. We find that the magnetic filling factor is related to the magnetic field strength by the simple law $\alpha=B_{1} / B$ with $B_{1}=15 \mathrm{G}$. This result is compatible with the Hanle weak-field determinations, as well as with the stronger field determinations from the Zeeman effect (kGauss field filling 1-2\% of space). From linear fits, we obtain the analytical dependence of the magnetic field PDF. Our analysis has also revealed that the magnetic field in the quiet Sun is isotropically distributed in direction.

Conclusions. We conclude that the quiet Sun is a complex medium where magnetic fields having different field strengths and filling factors coexist. Further observations with a better polarimetric accuracy are, however, needed to confirm the results obtained in the present work.
\end{abstract}

Key words. magnetic fields - polarization - turbulence - techniques: polarimetric - methods: data analysis - Sun: magnetic fields

\section{Introduction}

The solar magnetic field is divided into two classes: the network field, and the internetwork field. The network field is the one of sunspots and active regions (plages or faculae), but it appears also in quiet regions as pepper-and-salt grains scattered in longitudinal magnetograms, indicating a stronger field than in their surroundings. These grains delineate the frontiers of a network

\footnotetext{
* Based on observations made with the French-Italian telescope THEMIS operated by the CNRS and CNR on the island of Tenerife in the Spanish Observatorio del Teide of the Instituto de Astrofísica de Canarias.

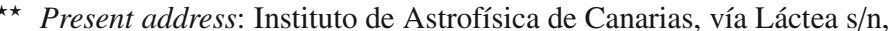
38205 La Laguna, Tenerife, Spain.
}

of so-called supergranules, each supergranule having a width of about $30000 \mathrm{~km}$. The internetwork field lies inside the supergranules. A map of spectropolarimetric data, including active and quiet regions (and a filament) was analyzed by Bommier et al. (2007) in terms of vector magnetic field, by applying the UNNOFIT inversion code. It was found that these two classes of magnetic fields are characterized by different field strengths and directions: while the network field is found rather vertical with a field strength of $100 \mathrm{G}$ or higher (spatially averaged), the internetwork is found to be far weaker (in spatial average) and turbulent in direction. In quiet regions, the solar magnetic field appears then as vertical "trees" standing in places (on the supergranules frontiers) out of the "carpet" of the turbulent internetwork field. 
However, the internetwork magnetic field had already been investigated. The first attempt to measure the internetwork field comes from Stenflo (1982), who established the first and founding results: some lines observed near the solar limb are found linearly polarized, and this polarization stems from radiative scattering near the surface, because the incident radiation is anisotropic due to limb darkening. But the observed polarization degree is found to be lower than the theoretical one in a pure scattering model. Thus, Stenflo was led to introduce a possible magnetic depolarization, the Hanle effect. Stenflo (1982) evaluates the order of magnitudes, resulting in the range $10-100 \mathrm{G}$ for the internetwork field strength. This order of magnitude was later confirmed by a detailed theoretical calculation by FaurobertScholl (1992). The Hanle effect is sensitive to the magnetic field strength when the Larmor pulsation $\omega$ is comparable to the upper-level inverse lifetime $\tau^{-1}$, i.e. when $\omega \tau \sim 1$. For permitted visible lines where $\tau \sim 10^{-7}-10^{-8} \mathrm{~s}$ (in the UV or IR domains, this order of magnitude differs), this leads to $B \sim 1-10 \mathrm{G}$. For 100 times higher field strengths, the Hanle effect saturates and only the sensitivity to the field direction remains. In the Hanle field range, the sensitivity of the Zeeman effect is weak, and the Hanle effect observed in visible lines is then revealed as the welladapted tool for the weak field measurements. Moreover, the rotation of the polarization direction, which would also be observed for the Hanle effect in a deterministic field, has remained undetectable, leading Stenflo to conclude that there is a turbulent field (in direction). As the Hanle effect is highly nonlinear, it is also well-adapted to the detection of a turbulent field, whereas the Zeeman effect would remain globally insensitive because it is linear.

Later on, however, strong $\mathrm{kG}$ fields were also detected in quiet regions (Grossmann-Doerth et al. 1996), on small scales, smaller than the resolution element. Thus, a magnetic filling factor $\alpha<1$ was to be introduced to interpret the observations, as earlier done in the network case (Stenflo 1973). And, time passing, the spatial resolution of both ground-based (VTT, THEMIS) and spaceborne (HINODE) instruments increased, raising the possibility of Zeeman detection of the weak fields. The IR window opened also, where the Zeeman effect is relatively more sensitive. We provide the references to the related new measurements later on in this paper. But, depending on the presence or absence of a magnetic filling factor in the models, the situation seems to us to be rather involved in discussions of the quiet Sun magnetic field probability density function (PDF), in particular with respect to its definition and evaluation.

From ground-based telescopes, the spatial resolution of spectro-polarimetric data is about $0.5-1^{\prime \prime}$. At this stage, the only reliable results concerning the magnetic field strength in the quiet Sun have been performed in the near-IR (Khomenko et al. 2003; Martínez González et al. 2008a) or by using spectral lines with hyperfine structure (López Ariste et al. 2006; Ramírez Vélez et al. 2008) or spectral lines sensitive to the Hanle effect (Trujillo Bueno et al. 2004, and Refs. therein). The studies using the visible Fe I $6301.5 \AA$ and $6302.5 \AA$ spectral lines, which show stronger field in the $\mathrm{kG}$ range (Socas-Navarro \& Sánchez Almeida 2002; Domínguez Cerdeña et al. 2003a; Socas-Navarro et al. 2004; Lites \& Socas-Navarro 2004), have been put in doubt (Martínez González et al. 2006; López Ariste et al. 2007; Khomenko \& Collados 2007).

Khomenko et al. (2003) and Martínez González et al. (2008a) have derived the PDF of the magnetic field on the quiet Sun using the $1.5 \mu \mathrm{m}$ Fe I spectral lines. The magnetic field strengths inferred by both analyses were in the hG regime, with values around the equipartition field in the photosphere. They obtained a magnetic field PDF that could be reproduced by a decreasing exponential law. This exponential form for the PDF was then used by Trujillo Bueno et al. $(2004,2006)$ to interpret second solar spectrum observations (from various authors and instruments) in terms of Hanle depolarization due to a turbulent field. From these observations, these authors propose that the mean magnetic field is $130 \mathrm{G}$.

The HINODE satellite has provided spectro-polarimetric data in the $6302.5 \AA$ spectral range with a spatial resolution of about $0.32^{\prime \prime}$. The validity of the analysis of this kind of data has been studied by Orozco Suárez et al. (2007a). The inversion of quiet Sun HINODE data has resulted in a PDF containing hG fields, as pointed out by the infrared measurements but in disagreement with the previous $6302.5 \AA$ studies mentioned above (Orozco Suárez et al. 2007b).

In this paper, we deal with $6302.5 \AA$ spectro-polarimetric data to infer the magnetic field PDF in the quiet Sun. The observations were taken with the ZIMPOL polarimeter mounted on the THEMIS telescope and are described in Sect. 2. To overcome the difficulties with the $6302.5 \AA$ interpretation, we propose an analysis procedure that combines the results of the UNNOFIT inversion code (first step, determination of the local average field, Sect. 3) and direct observables of the polarized profiles (second step, direct determination of the magnetic filling factor, Sect. 4). It is then possible to derive the PDF (Sect. 5), but we had to return to the basic statistical definitions to clarify the role played by the magnetic filling factor in the PDF definition (see also Appendix A). In Sect. 5 we compare our results with the previous ones, which leads us to clarify the notion of PDF applied to the quiet Sun magnetic field and to reexamine the validity conditions of the Fe I $6302.5 \AA$ inversion. Our analysis is compatible with previous quiet Sun studies, revealing that the greater the magnetic field strength the less the filling factor. We provide analytical fits of our histograms, leading to an analytical PDF.

Our aim in writing this paper is to get a statistical approach to the quiet Sun magnetic field and to disentangle the notions of magnetic field strength distribution and filling factor. Our main contribution is to directly determine the magnetic filling factor, which is independent of the data inversion.

\section{Observations}

The observations were performed on the 5 and 6 July 2008, with the ZIMPOL polarimeter mounted on the THEMIS telescope (ZIMPOL II, see Gandorfer \& Povel 1997). The aim of these observations was to investigate the quiet Sun magnetic field at different atmospheric layers. In this prospect, two ZIMPOL cameras were installed, one observing the CrI $5781.8 \AA$ line (line center formation height $85 \mathrm{~km}$ ), and the other one observing the Fe I $6301.5 \AA$ and $6302.5 \AA$ lines (line center formation height $330 \mathrm{~km}$ and $260 \mathrm{~km}$, respectively). We recall that the ZIMPOL system requires one unmasked pixel every four pixels of the camera chip. The camera centered on the $6302 \AA$ spectral range was equipped with a microlens system that focuses the solar light in the unmasked pixel. The $5782 \AA$ camera was not equipped with this system. The integration time was thus significantly shorter for the $6302 \AA$ camera. The unmasked pixelsize was 0.13 arcsec, but the observation pixelsize results in 0.53 arcsec because of the microlens system. The spectral pixel was $7.18 \mathrm{~m} \AA$. The slit width was 0.5 arcsec.

The observations were performed with the slit fixed at disk center. The disk center was quiet. The slit was oriented solar 
Table 1. Information on the averaged data. Each line of the table corresponds to one average.

\begin{tabular}{ccccc}
\hline \hline Date & $\begin{array}{c}\text { Number of } \\
\text { images }\end{array}$ & $\begin{array}{c}\text { Number of } \\
\text { accumulations } \\
\text { per image }\end{array}$ & $\begin{array}{c}\text { Polarimetric } \\
\text { accuracy }\end{array}$ & $\begin{array}{c}\text { Total } \\
\text { duration } \\
(s)\end{array}$ \\
\hline 5.7 .2008 & 10 & 16 & $2.5 \mathrm{E}-04$ & 189 \\
5.7 .2008 & 8 & 16 & $2.8 \mathrm{E}-04$ & 151 \\
5.7 .2008 & 8 & 16 & $2.8 \mathrm{E}-04$ & 151 \\
5.7 .2008 & 21 & 16 & $1.7 \mathrm{E}-04$ & 397 \\
5.7 .2008 & 21 & 16 & $1.7 \mathrm{E}-04$ & 397 \\
5.7 .2008 & 9 & 16 & $2.6 \mathrm{E}-04$ & 170 \\
5.7 .2008 & 25 & 16 & $1.6 \mathrm{E}-04$ & 473 \\
5.7 .2008 & 14 & 16 & $2.1 \mathrm{E}-04$ & 265 \\
5.7 .2008 & 19 & 16 & $1.8 \mathrm{E}-04$ & 359 \\
6.7 .2008 & 15 & 32 & $1.4 \mathrm{E}-04$ & 500 \\
6.7 .2008 & 15 & 32 & $1.4 \mathrm{E}-04$ & 500 \\
6.7 .2008 & 15 & 32 & $1.4 \mathrm{E}-04$ & 500 \\
\hline
\end{tabular}

north. The exposure time was $320 \mathrm{~ms}$ (per accumulation), but we determined that 10 accumulations were needed to get a polarimetric accuracy of $1 \times 10^{-3}$. Due to the actuation (camera readout, analyzer plates rotation), the total duration was $0.9 \mathrm{~s}$ per accumulation. Several accumulations were averaged before being stored on the disk, the storage process taking about $4.5 \mathrm{~s}$. Actually, the polarimetric accuracy of $1 \times 10^{-3}$ (for 10 accumulations) was insufficient to get a signal in linear polarization, so we were obliged to average series of images. The polarimetric accuracy level was derived from the noise level along the observed profiles. The detail of the observations (number of averaged images, resulting polarimetric accuracy, total duration) is given in Table 1 . On 5 July the TIP-TILT stabilization system was ON, but some jumps occurred and we averaged the images between the jumps. On 6 July the TIP-TILT stabilization system was OFF but the seeing was excellent. We averaged the data by series of 15 images. Such a series corresponds to a total duration of less than $600 \mathrm{~s}$. We retained this limit because we observed that the linear polarization signal-to-noise ratio decreases if the total integration time is longer than the typical granule lifetime, which is around $600 \mathrm{~s}$. When the TIP-TILT was OFF, no alternative correction was applied for the seeing effect. We thus got a total of 12 different observations of the 140 pixels slit, which results in 1680 observed profiles on which we can perform a statistical analysis.

The magnetic field values given in the present paper were derived from the Fe I $6302.5 \AA$ observations. The ZIMPOL data reduction package was used (Gandorfer et al. 2004).

\subsection{Evaluation of the line center height of formation}

The temperature, electron pressure and gas pressure were taken from the Maltby et al. quiet Sun photospheric reference model (Maltby et al. 1986), extrapolated downwards beyond $-70 \mathrm{~km}$ to $-450 \mathrm{~km}$ below the $\tau_{5000}=1$ level. Above $-70 \mathrm{~km}$, this model is very similar to the quiet Sun VAL C (Vernazza et al. 1981). The continuum absorption coefficient was evaluated as in the MALIP code of Landi Degl'Innocenti (1976), i.e. by including $\mathrm{H}^{-}$bound-free, $\mathrm{H}^{-}$free-free, neutral hydrogen atom opacity, Rayleigh scattering on $\mathrm{H}$ atoms and Thompson scattering on free electrons. The line absorption coefficient was derived from the Boltzmann and Saha equilibrium laws, taking the two first ions of iron into account. The atomic data were taken from Wiese or Moore and the partition functions from Wittmann. The iron abundance was assumed to be 7.60 (in the usual logarithmic scale where the abundance of hydrogen is 12). A depth-independent microturbulent velocity field of $1 \mathrm{~km} \mathrm{~s}^{-1}$ was introduced. Finally, for layers above $\tau_{5000}=0.1$, departures from LTE in the ionization equilibrium were simulated by applying Saha's law with a constant "radiation temperature" of $5100 \mathrm{~K}$ instead of the electron temperature provided by the atmosphere model.

To get the result, the line center optical depth grid was scaled to the continuum one, by using their respective absorption coefficients. The continuum optical depth grid was the one provided with the atmosphere model and the transfer equation was not explicitely solved again. The height of formation of the line center (the one given above) was then determined as follows. Given the grid of line center optical depths, the height of formation of the line center is the one for which the optical depth along the line of sight is unity (Eddington-Barbier approximation), i.e. the one for which $\tau / \mu=1$, where $\tau$ is the line center optical depth along the vertical, and $\mu$ the cosine of the heliocentric angle $\theta$ (here taken at 0 ).

\section{First step: full Stokes inversion}

\subsection{Results on the quiet Sun magnetic field strength and direction}

The magnetic field vector was determined by applying the UNNOFIT inversion (Bommier et al. 2007; Landolfi et al. 1984), which is based on the Milne-Eddington approximation. A 2-component atmosphere is assumed: one magnetic (with filling factor $\alpha$ ) and one non-magnetic (with filling factor $1-\alpha$ ). These two atmospheres are assumed to have all their other parameters identical, except for the presence/absence of the magnetic field. Only the information of the $6302.5 \AA$ spectral line is taken into account. The four Stokes parameters are inverted simultaneously. An example of observed profile and the best fit obtained with UNNOFIT is shown in Fig. 1. In the examination of the differences between the theoretical and observed profiles in this figure, it can first be pointed out that the noise level, measurable in the continuum, is non negligible with respect to the signal order of magnitude, especially in $Q / I$ and $U / I$. Second, it has to be considered that the four Stokes profiles are simultaneously fitted, by using a rather simple atmosphere model (MilneEddington): thus, departures may not be surprising. However, we find that the theoretical profile shape corresponds to the observed one, in terms of sign and components. Thus we conclude that, under the ME hypothesis, we are able to retrieve the field vector within an uncertainty that is clarified in the following.

The neighboring Fe I 6301.5 A line was also observed, but its inversion fails in a number of pixels that we estimate too high. This comes from its lower sensitivity to the magnetic field: first because its Landé factors are smaller, and second because it is not a normal Zeeman triplet line $(J=1 \leftrightarrow J=0)$ contrary to 6302.5 . Its Zeeman polarization is more entangled along the spectrum, resulting in a lower global sensitivity. We applied UNNOFIT2, the inversion code adapted to lines that are not normal Zeeman triplet and got this unsatisfactory result. Besides, both lines 6302.5 and 6301.5 could be inverted together, but as pointed out by Martínez González et al. (2006), they are not formed at the same depth, on the one hand, and one line is enough for Milne-Eddington inversion in terms of number of researched parameters, on the other.

As shown by Bommier et al. (2007), the 2-component inversion of Fe I $6302.5 \AA$ is unable to separately determine the magnetic filling factor $\alpha$ and the magnetic field strength $B$, but 

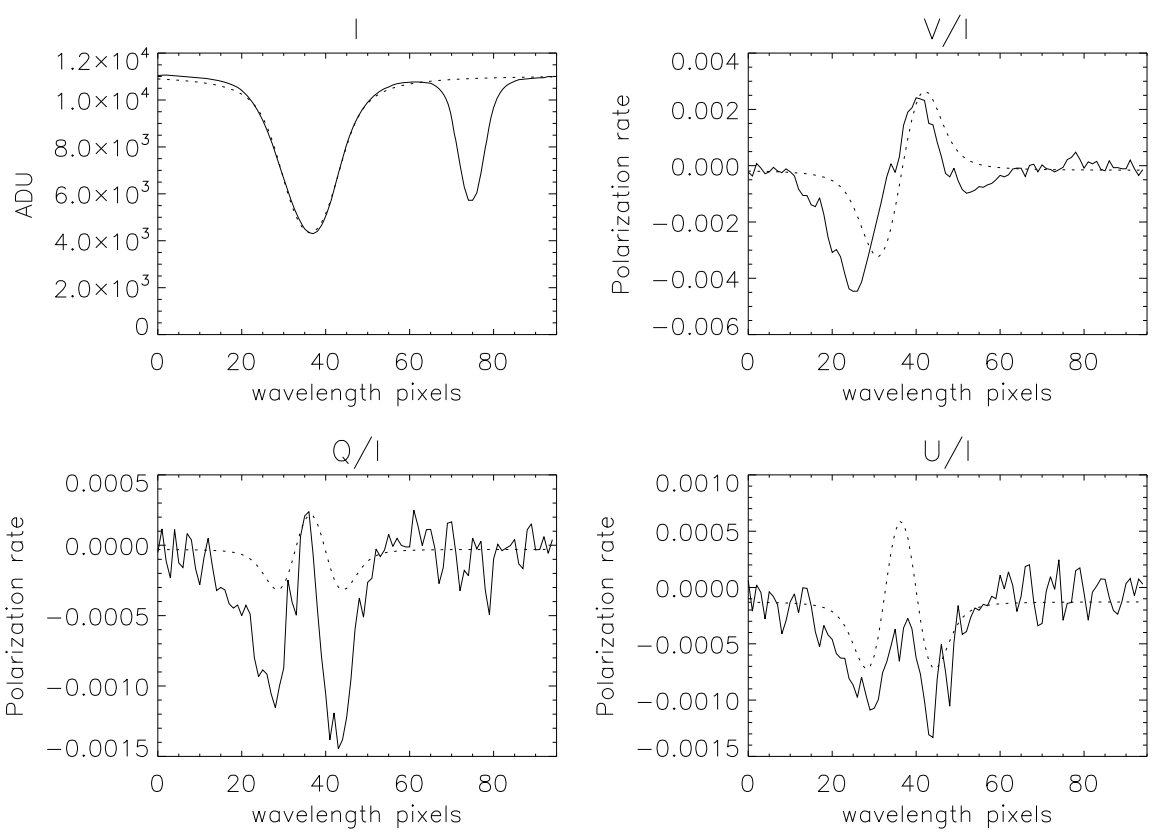

Fig. 1. UNNOFIT fit (dotted line) of a typical observation (full line). The main spectral line (centered at pixel 38), that is polarized, is Fe I $6302.5 \AA$, and the other line, unpolarized, is a telluric line. 10 pixels are $72 \mathrm{~m} \AA$ and blue is towards the left. Derived magnetic field parameters: local average field strength $\alpha B 11.7 \mathrm{G}$, inclination $115^{\circ}$ from the line-of-sight, azimuth $123^{\circ}$ from the slit direction which is the reference axis for the Stokes parameters. The line strength $\eta_{0}$ parameter is found to be 2.6, and the Doppler width $35.8 \mathrm{~m} \AA$.

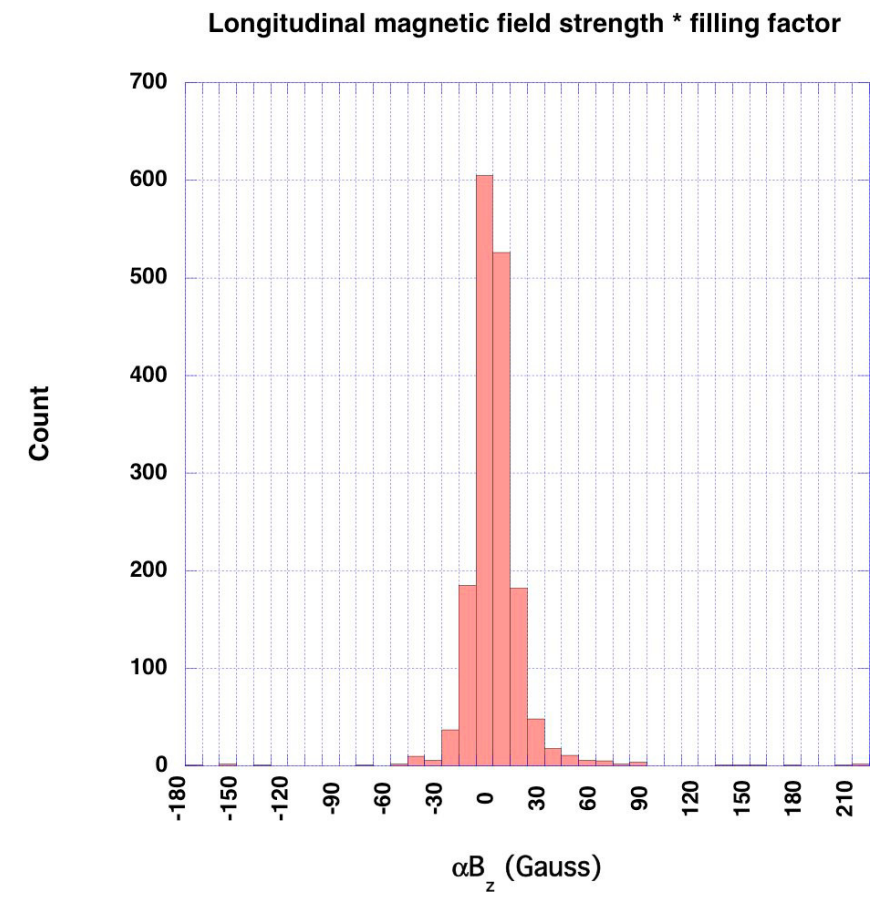

Fig. 2. Histogram of the local average longitudinal magnetic field (the product of the longitudinal magnetic field $B_{z}$ with the magnetic filling factor $\alpha$ ), from the UNNOFIT inversion.

only their product $\alpha B$, which we call the "local average magnetic field strength". Figures 2-5 display histograms constructed from the UNNOFIT inversion of the 1680 observed profiles. Figure 2 displays the histogram of the local average longitudinal magnegtic field $\alpha B_{z}$, which is the magnetic flux. The unsigned average magnetic flux is found to be $11 \mathrm{Mx} / \mathrm{cm}^{2}$. Figure 3 displays the histogram of the local average magnetic field strength $\alpha B$ for all the observed profiles. The most probable value is $\alpha B=13 \mathrm{G}$, the probability for stronger fields decreasing very fast. The mean value is $\alpha B=18 \mathrm{G}$ and the standard deviation is $14 \mathrm{G}$.

Figure 4 displays the histogram of the magnetic field vector inclination. First we recall that an isotropic distribution of field

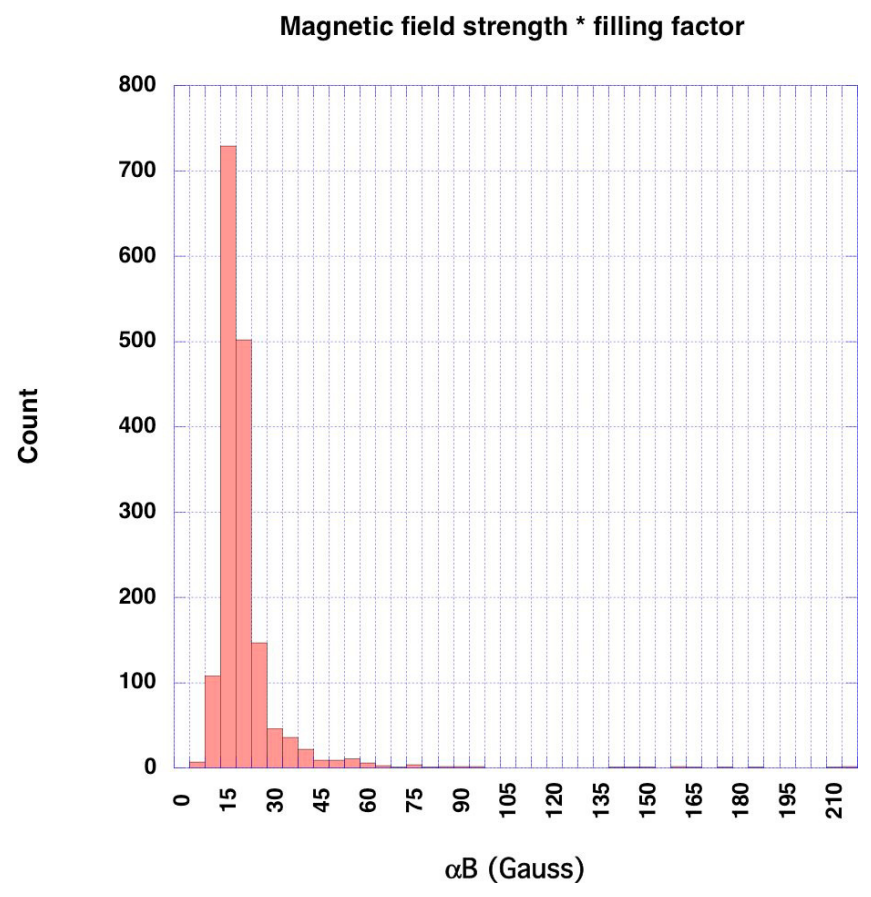

Fig. 3. Histogram of the local average magnetic field strength (the product of the magnetic field strength $B$ with the magnetic filling factor $\alpha$ ), from the UNNOFIT inversion.

directions leads to a histogram for the inclination that has a sinusoidal shape because the elementary surface on the unit sphere is $\sin \theta \mathrm{d} \theta \mathrm{d} \varphi$. Such a shape was obtained in previous THEMIS observations (Bommier et al. 2007), but it was wrongly concluded that the magnetic field tends to be horizontal because the inclination angles were also predominantly ranging between $35^{\circ}$ and $145^{\circ}$. Disregarding the central hollow, the histogram in Fig. 4 displays the sinusoidal shape expected for the inclination angles of an isotropic distribution. This central void is the result of the presence of $V$ profiles in all the observed pixels. Simultaneous non-zero $Q, U$ and $V$ is not surprising if many magnetic fields with different inclinations actually coexist in each resolution 


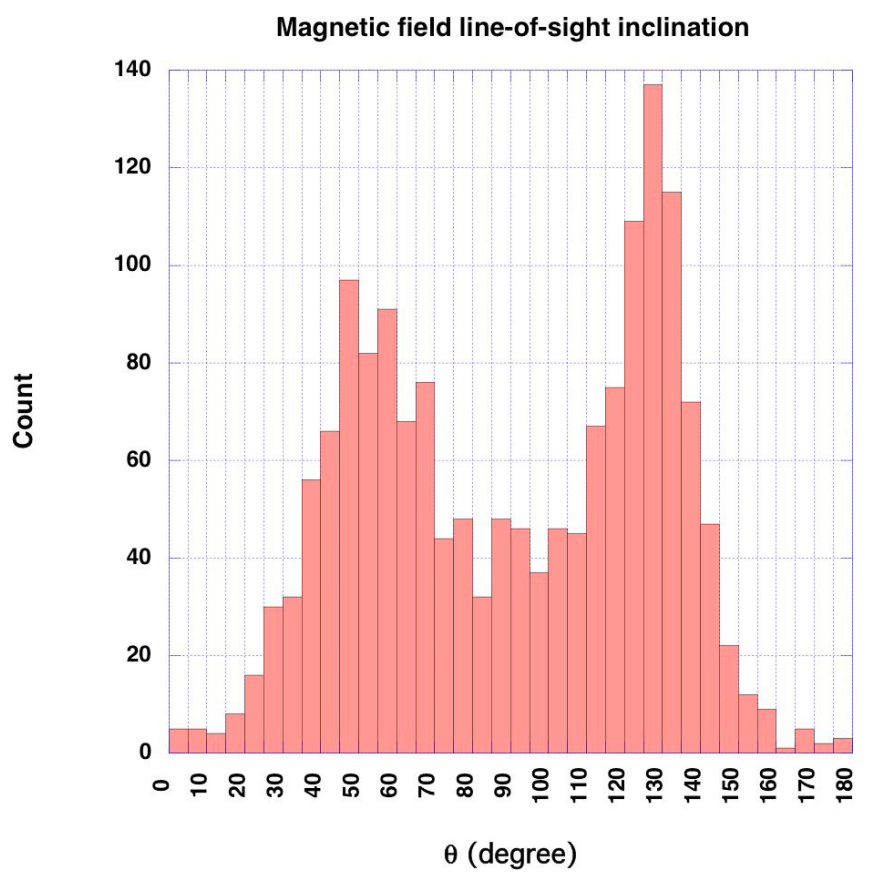

Fig. 4. Histogram of the magnetic field inclination with respect to the line-of-sight, from the UNNOFIT inversion. The line-of-sight is also the solar vertical with the observation performed at disk center.

element. The central hollow could stem from our modeling by a single magnetic field per element. Another explanation for the presence of Stokes $V$ could be some misalignment problem in the data reduction. We note here that the inclination histogram of HINODE data by Ishikawa \& Tsuneta (2009, see their Fig. 5) also displays a central hollow, although not as marked as here. From the accuracy test described further, the inclination angle is determined within $\pm 10^{\circ}$. Considering also the small average number of analyzed profiles per bin (see the discussion in the next paragraph), it is impossible to ascertain a departure from the isotropic distribution in the last four bins at the extremities where the envelope tangent seems to be horizontal, in contrast to a $\sin \theta$ envelope.

Figure 5 displays the histogram of the azimuth, which is defined with respect to the slit direction. As the fundamental $\left(180^{\circ}\right)$ ambiguity is not resolved, the azimuth is defined modulo $\pi$ between $0^{\circ}$ and $180^{\circ}$. This histogram displays a flat shape on average, also corresponding to the azimuths of an isotropic distribution. More precisely, the average number of counts per bin is about $N \simeq 40$. Assuming a Gaussian noise, the noise level per bin is $\sqrt{N} \simeq 6.5$. In the figure, the deviations from bin to bin generally agree with this value, except in some cases where it is higher. In these cases a positive deviation is, however, most often immediately followed by a negative one of the same order of magntitude, and there is no case where this deviation is greater than $3 \sqrt{N} \simeq 20$, which would be really significant. Thus, we conclude that in a first approximation our observations indicate an isotropic distribution of the quiet Sun magnetic field azimuths.

We conclude from the THEMIS observations (Bommier et al. 2007) and from these new ZIMPOL observations that the quiet Sun magnetic field has most likely an isotropic distribution of directions. Observations performed at different limb distances by Martínez González et al. (2008b) also conclude on an isotropic distribution of the quiet Sun magnetic field direction. Such observations at different limb distances are indeed

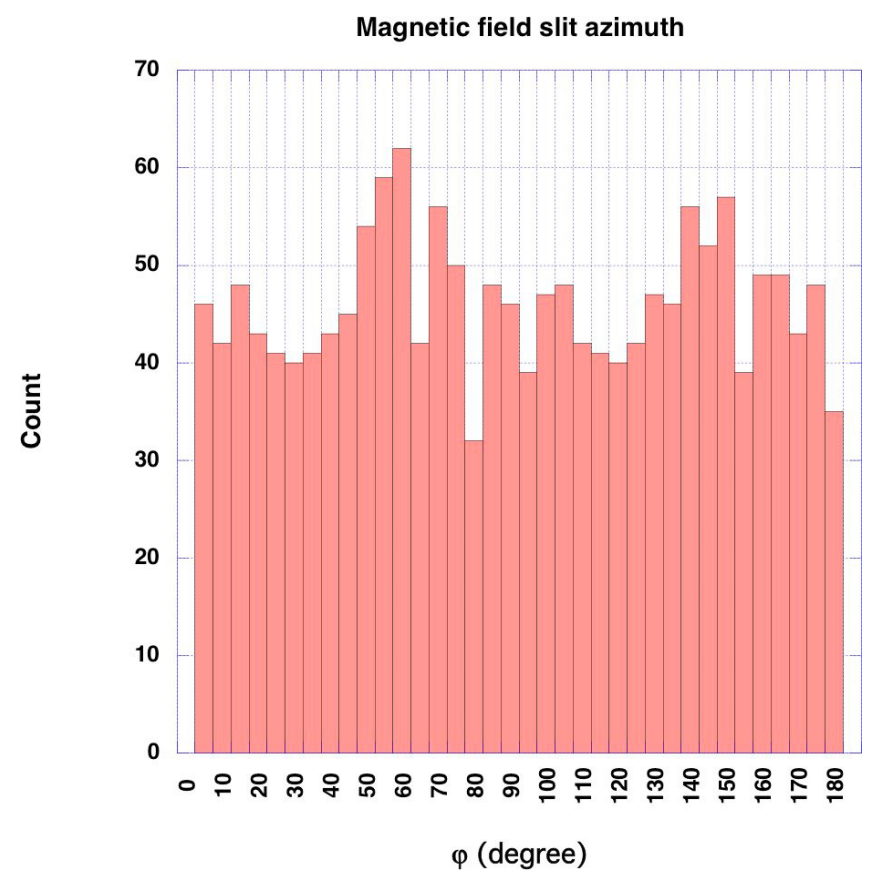

Fig. 5. Histogram of the magnetic field azimuth with respect to the slit direction, from the UNNOFIT inversion (ambiguity is not resolved). The observation was performed at disk center and the slit was solar north, so that this is also the histogram of the horizontal field component azimuth.

needed to thoroughly investigate the field direction distribution. We performed them with ZIMPOL on THEMIS, and we shall discuss them in a future paper of this series. On the basis of our present observations, we do not confirm the horizontal trend of the internetwork magnetic field recently observed by HINODE (Lites et al. 2007, 2008) and derived from HINODE inclination histograms by Orozco Suárez et al. (2007b) and Ishikawa \& Tsuneta (2009), who could have also been unaware of the sinusoidal shape of the inclination histogram from an isotropical distribution.

\subsection{Accuracy of the inversion}

To determine the accuracy of the magnetic inversion, we performed the same test as in Bommier et al. (2007), but for the ZIMPOL/THEMIS polarimetric accuracy $\left(2 \times 10^{-4}\right.$ in the continuum under the conditions described in Sect. 2) and spectral sampling. As in that paper, a series of theoretical profiles was generated from the Unno-Rachkovsky solution applied to the 2-component atmosphere, for a set of magnetic field strength, inclination, azimuth, and filling factor values. These profiles were then noised at the observed level and submitted to the UNNOFIT inversion. The test consists in comparing the output values with the known input ones. The main result is presented in Fig. 6, which is analogous to Fig. 4 of Bommier et al. (2007), and it shows analogously that the local average magnetic field strength $\alpha B$ (the product of the field strength by the filling factor) is correctly determined by the inversion. The bottom figure is a zoom of the top figure near the axis origin, and shows the dispersion of the results about the first diagonal that represents equal input and output. This dispersion is on the order of $10 \mathrm{G}$, which we retain as the accuracy on the local average magnetic field strength determination. This dispersion includes both longitudinal and transverse fields. Moreover, the bottom figure shows that, 

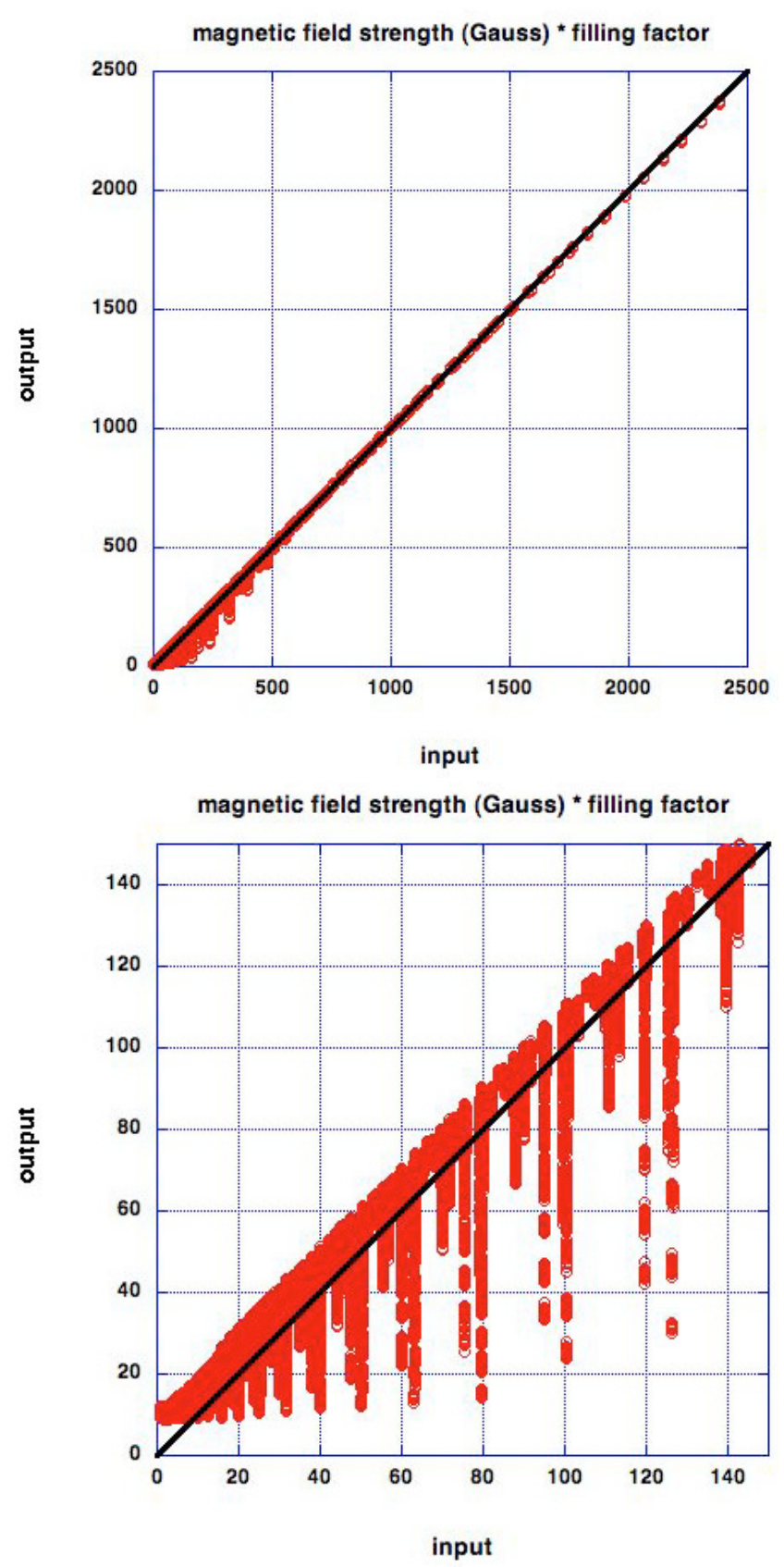

Fig. 6. Test of the determination of the local average magnetic field strength (the product of the magnetic field strength $B$ with the magnetic filling factor $\alpha$ ) by the UNNOFIT inversion. A series of theoretical profiles are computed under the conditions of the ZIMPOL observations (spectral and polarimetric resolutions), and then submitted to the inverter. In abscissae are the input known values, in ordonnae are the output recovered values. The bottom figure is a zoom of the top figure near the axis origin.

when the input local average magnetic field strength decreases to zero, the output saturates at $10 \mathrm{G}$. In Fig. 4 of Bommier et al. (2007), the saturation level is $25 \mathrm{G}$ for a polarimetric accuracy of only $1.5 \times 10^{-3}$. It thus appears that the saturation level is directly related to the polarimetric accuracy. The regular pattern detectable in the lower part of Fig. 6 is a consequence of the regular spacing of the input theoretical data. The number of input points is 183600 and the number of output points that depart from more than about $10 \mathrm{G}$ from the diagonal is about $5 \%$ of the total number of points.

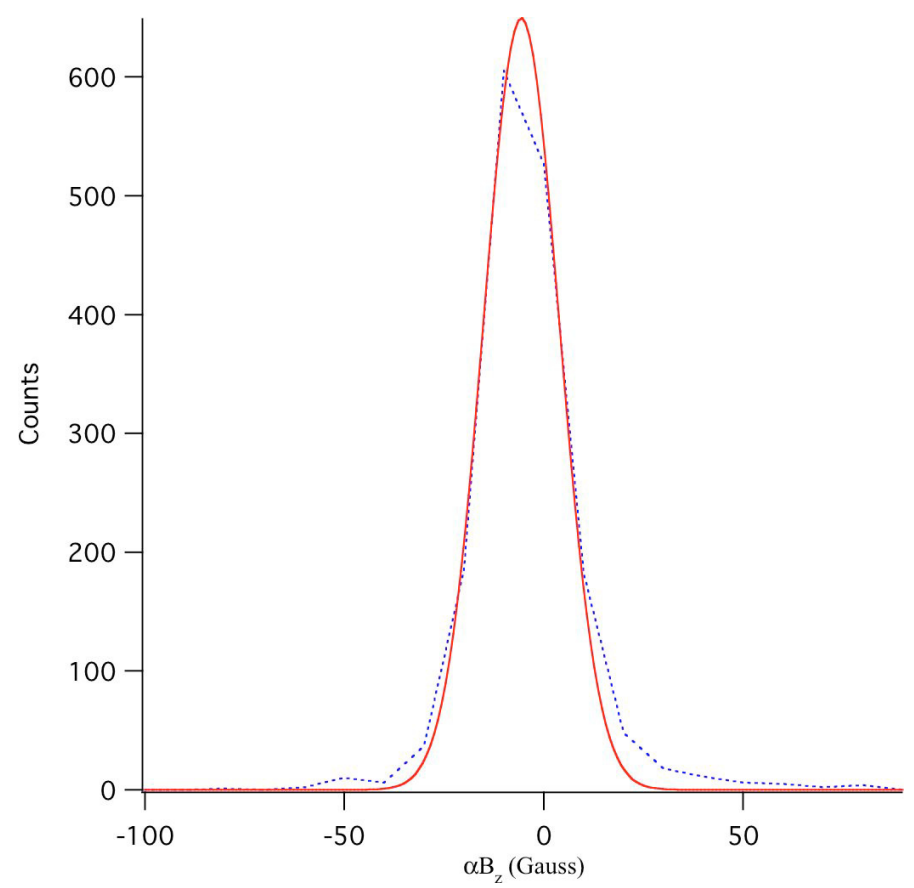

Fig. 7. Fit of the local average longitudinal magnetic field histogram of Fig. 2 by a Gaussian $\exp \left(-\left(\left(x-x_{0}\right) / w\right)^{2}\right)$ of half-width $w=13.5 \mathrm{G}$.

The observed profile asymmetries, which are visible in Fig. 1, are taken into account neither in the test nor in the inversion. Such asymmetries may be due to vertical gradient of the radial velocity. A new version of our inversion code UNNOFIT is under development, which takes into account such gradients and is able to properly fit asymmetric profiles. At first sight the magnetic field strength would not be highly modified. Besides, it may be noted that the asymmetries' order of magnitude is comparable to the polarimetric noise in Fig. 1, at least in $Q / I$ and $U / I$.

Figures 7 and 8 display Gaussian fits of the histograms of Figs. 2 and 3, corresponding respectively to the local average longitudinal magnetic field and to the local average magnetic field strength. The histogram of Fig. 2 can be fitted with the usual Gaussian $\exp \left(-\left(\left(x-x_{0}\right) / w\right)^{2}\right)$. The histogram in Fig. 3 can be fitted by the Maxwell distribution $4 \pi x^{2} \exp \left(-(x / w)^{2}\right)$. These fits show that the magnetic field vector has a Gaussian distribution, since a Gaussian distribution leads to a Maxwell distribution after angle averaging. The width $w$ of the Gaussian and Maxwellian are $13.5 \mathrm{G}$ and $11 \mathrm{G}$, respectively. Since these values are not significantly higher than the $10 \mathrm{G}$ corresponding to the inversion accuracy, we cannot conclude that the local average magnetic field truly has a Gaussian PDF, so we attribute the Gaussian shape of these histogram envelopes to the polarimetric noise.

\section{Second step: direct determination of the magnetic filling factor}

From the weak field laws, which express the emerging polarization Stokes parameters in terms of the derivatives of the intensity profile, Landi Degl'Innocenti \& Landolfi (2004, pp. 405-407) derive the approximate expressions

$\frac{|V|_{\max }}{I_{\mathrm{c}}} \simeq \alpha \frac{\Delta \lambda_{\mathrm{B}}}{\Delta \lambda_{\mathrm{D}}} \cos \theta \bar{g} d_{\mathrm{c}}$ 


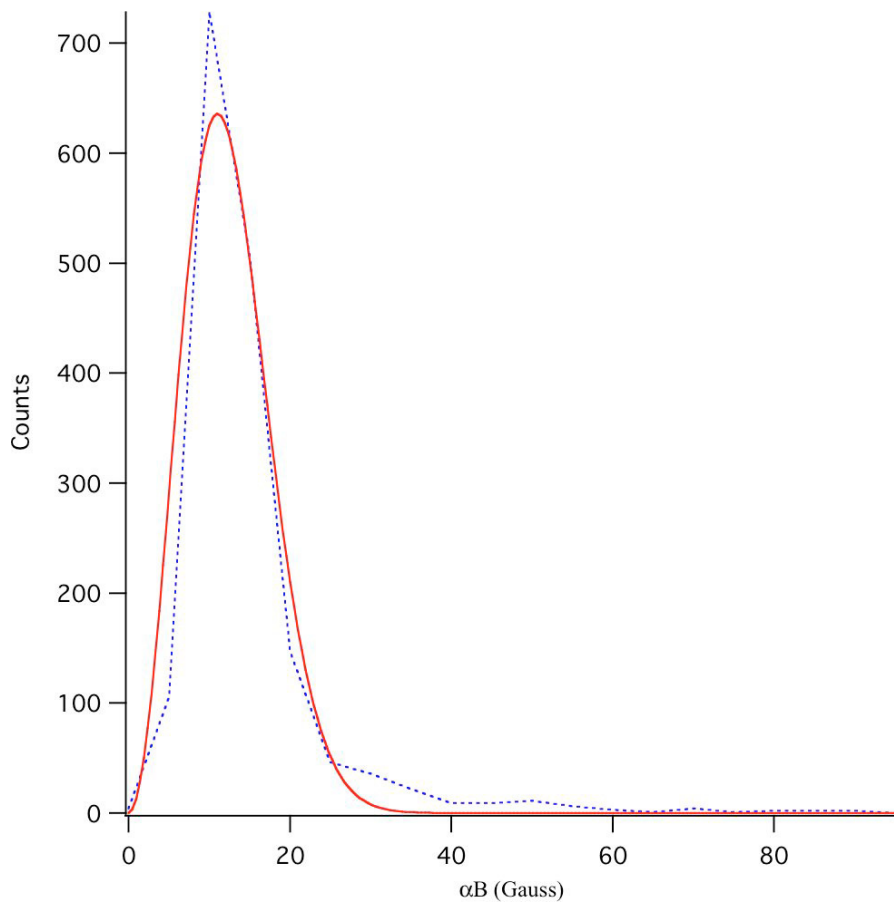

Fig. 8. Fit of the local average magnetic field strength histogram of Fig. 3 by a Maxwellian $4 \pi x^{2} \exp \left(-(x / w)^{2}\right)$ of half-width $w=11 \mathrm{G}$.

$\frac{\left(\sqrt{Q^{2}+U^{2}}\right)_{\max }}{I_{\mathrm{c}}} \simeq \alpha\left(\frac{\Delta \lambda_{\mathrm{B}}}{\Delta \lambda_{\mathrm{D}}}\right)^{2} \sin ^{2} \theta \bar{G} d_{\mathrm{c}}$.

We have introduced here the magnetic filling factor $\alpha$, which is not in Landi Degl'Innocenti \& Landolfi (2004, pp. 405-407). Here, $\Delta \lambda_{\mathrm{D}}$ is the Doppler width and $\theta$ the magnetic field vector inclination with respect to the line-of-sight. $\Delta \lambda_{\mathrm{B}}$ is the Zeeman splitting in wavelength units, defined by

$\Delta \lambda_{\mathrm{B}}=\lambda_{0}^{2} \frac{\nu_{\mathrm{L}}}{c}$,

where $v_{\mathrm{L}}$ is the Larmor frequency (Landi Degl'Innocenti \& Landolfi 2004, p. 78). In numerical values, this is

$\Delta \lambda_{\mathrm{B}}=4.6686 \times 10^{-10} \lambda_{0}^{2} B$,

where $\Delta \lambda_{\mathrm{B}}$ is in $\mathrm{m} \AA, \lambda_{0}$ is in $\AA$ and $B$ in $\mathrm{G} . \bar{g}$ is the effective Landé factor, and $\bar{G}$ is the second order effective Landé factor introduced by Landi Degl'Innocenti \& Landolfi (2004), that is equal to $\bar{g}^{2}$ in the case of Zeeman triplet lines like Fe I $6302.5 \AA$. $d_{\mathrm{c}}$ is the line depth

$d_{\mathrm{c}}=\frac{I_{\mathrm{c}}-I_{0}}{I_{\mathrm{c}}}$

where $I_{\mathrm{c}}$ is the continuum intensity near the line and $I_{0}$ is the line center intensity.

We now note that the linear polarization depends quadratically on the magnetic field strength and direction, but linearly on the magnetic filling factor. Taking the square of Eq. (1) and dividing by Eq. (2), we find

$\alpha \simeq \frac{|V|_{\max }^{2} \tan ^{2} \theta}{\left(\sqrt{Q^{2}+U^{2}}\right)_{\max }\left(I_{\mathrm{c}}-I_{0}\right)}$.

Figure 9 displays a test of this approximation. The Stokes parameters are first computed with the Unno-Rachkovsky solution applied to a 2-component atmosphere with a given value
Test $\alpha$

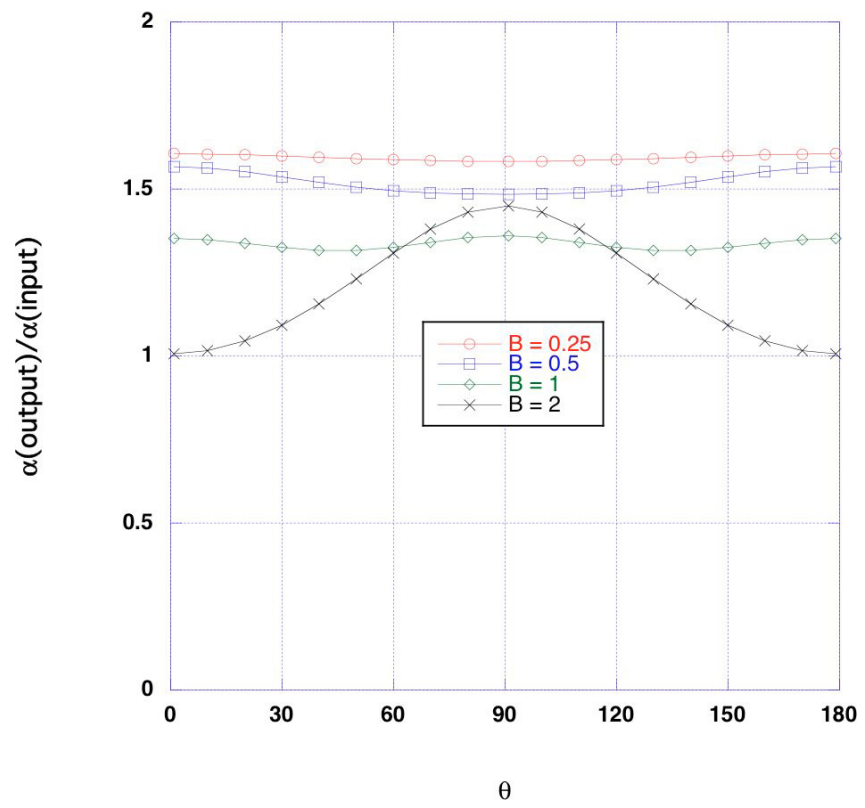

Fig. 9. Test of the determination of the magnetic filling factor by applying Eq. (6) to the polarimetric data. For the test, the polarimetric data were theoretical profiles computed from the Unno-Rachkovsky solution, weighted by a theoretical input magnetic filling factor $\alpha$ (input). Equation (6) is then applied, leading to $\alpha$ (output). The figure displays their ratio, for a series of magnetic field inclination angles and strengths (in Doppler width units). This plot is valid for any $\alpha$ value.

of $\alpha$ ( $\alpha$ input). The other parameters needed to compute the line profile are taken from the average values resulting from the UNNOFIT inversion applied to our Fe I $6302.5 \AA$ data. In particular the line strength is $\eta_{0}=2$. The output values of $\alpha$ are computed with Eq. (6). Figure 9 was plotted for a series of magnetic field strengths and inclinations. The field strengths are given in Doppler width units $\bar{g} \Delta \lambda_{\mathrm{B}} / \Delta \lambda_{\mathrm{D}}$. The different values 0.25 , $0.50,1$, and 2 Doppler widths correspond to $236,472,944$, and $1888 \mathrm{G}$, respectively, in the case of the Fe I $6302.5 \AA$ line formed in the quiet Sun. This figure is the same for any input $\alpha$ value.

In Fig. 10, we plotted the histogram derived from the application of Eq. (6) to our data. The inclination angle value $\theta$ was taken from the UNNOFIT inversion results. We thus obtain a filling factor $\alpha$ ranging between $3 \times 10^{-3}(\log \alpha=-2.5)$ and $1(\log \alpha=0)$, with a maximum probability at $8 \times 10^{-3}(\log \alpha=$ $-2.1)$. The mean value is $1.5 \times 10^{-2}(\log \alpha=-1.8)$. The standard deviation of $\log \alpha$ is 0.5 . The values higher than unity have no physical meaning and come from the fact that for a quasihorizontal field, when $\theta$ is close to $90^{\circ}, \tan \theta$ becomes very large.

From the polarimetric accuracy $\Delta S=2 \times 10^{-4} I_{\mathrm{c}}$ of our ZIMPOL/THEMIS data ( $S$ being any of the Stokes parameters $I, Q, U, V)$, we find that the relative error predicted by Eq. (6) is

$\frac{\Delta \alpha}{\alpha}=\frac{2 \Delta S}{|V|_{\max }}+\frac{\Delta S}{\left(\sqrt{Q^{2}+U^{2}}\right)_{\max }} \approx 0.5$.

Because $\log 0.5=-0.3$, we find that the inaccuracy is not negligible but nevertheless lower than the standard deviation of the $\alpha$ values displayed in the histogram of Fig. 10. We conclude that the dispersion we observe has a physical meaning, but that further observations with better polarimetric accuracy are needed to confirm this result. 


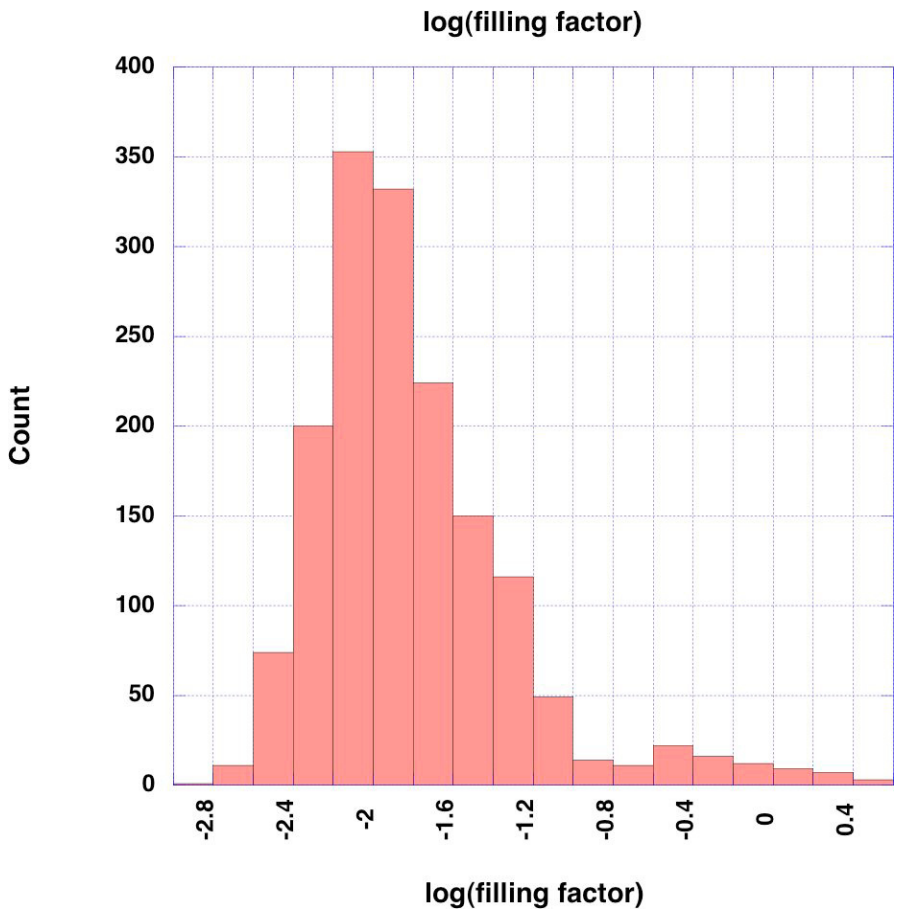

Fig. 10. Histogram of the filling factor, determined from the polarization data complemented by the UNNOFIT inversion results on the field inclination (see Eq. (6)). The abscissa is in logarithmic scale.

\section{The magnetic field probability density function}

For each of the 1680 observed solar pixels (determined by the slit width and the camera pixel size along the slit), the local average magnetic field strength $\alpha B$ was obtained from the UNNOFIT inversion (Sect. 3), and the magnetic filling factor $\alpha$ was directly and independently determined from the spectropolarimetric data (Sect. 4). The magnetic field strength value is then obtained by performing the ratio $\alpha B / \alpha$ for each pixel, and the magnetic field strength $B$ histogram follows (Fig. 11). The PDF of the magnetic field strength is then the envelope of this histogram. The tail of very strong fields that appears in the histogram stems from the noise contribution, but detecting strong fields associated to small filling factors is not new (see for instance Grossmann-Doerth et al. 1996; Socas-Navarro \& Sánchez Almeida 2002; Domínguez Cerdeña et al. 2003a,b). Khomenko et al. (2003) obtained a $B$ histogram similar in shape to ours, but with lower field strengths. Their histogram is devoid of any filling factor effect, because it results from direct Zeeman splitting measurement in IR profiles, where the Zeeman effect is stronger. However, as clearly shown by their Figs. 2 and 4, the Zeeman components are not yet completely resolved (in the near IR range), so that the real situation is probably inbetween the weak field approximation described by our Eq. (1) where the component separation does not depend on the field strength but instead on the derivative of the intensity profile and the completely resolved Zeeman effect. This circumstance could explain the lowest field strengths reported by these authors.

Thus, for each pixel, we find one $\alpha$ and one $B$ value. These values can be used to place a point representing the pixel in the $\log \alpha-\log B$ axes, thus giving a scatter plot where all the 1680 pixels are represented (Fig. 12). We find that these data are well-fitted (in the log-log coordinates) by the linear function

$\log \alpha=1.18-\log B$

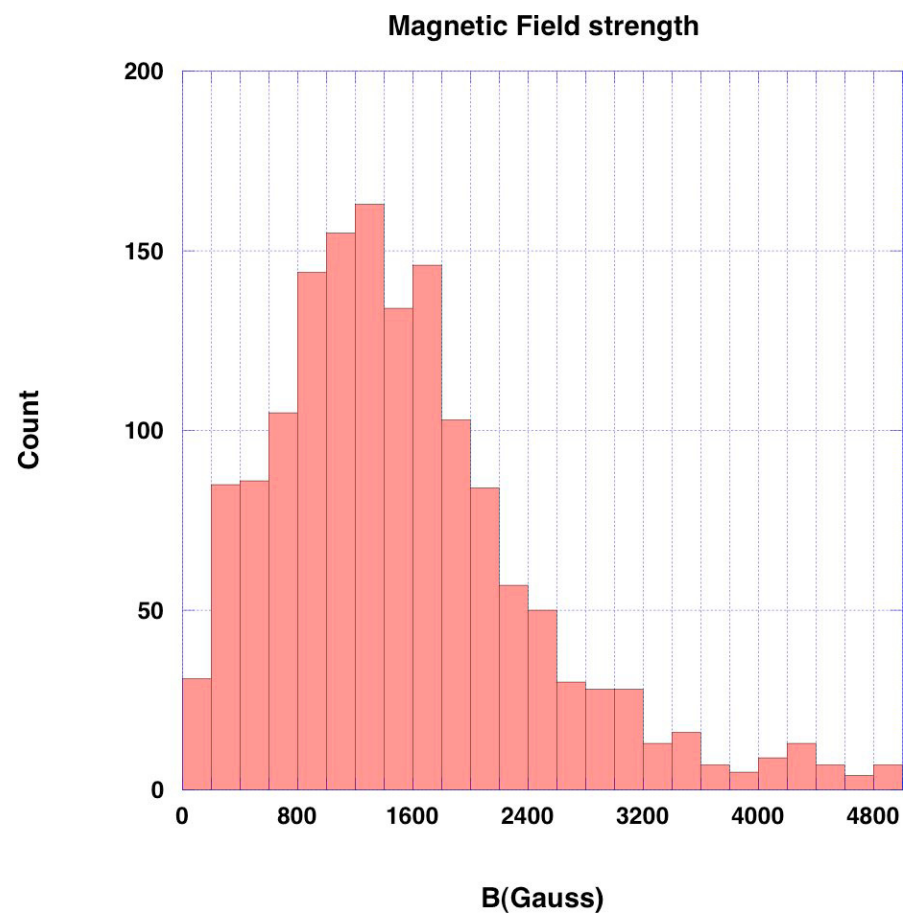

Fig. 11. Histogram of the magnetic field strength, derived by dividing the local average magnetic field strength $\alpha B$ by the independently determined magnetic filling factor $\alpha$, in each solar pixel. The envelope of this histogram is the magnetic field probability density function (PDF), apart from a normalization factor. Actually, this PDF is a marginal density function of the joint density function $p(\alpha, B)$ of the two random variables $\alpha$ and $B$, represented in Fig. 13. The abscissa is on a linear scale.

which is

$\alpha=\frac{B_{1}}{B}$

with $B_{1}=15 \mathrm{G}$. The form of this very simple relation partly comes from the $\alpha B$ histogram of Fig. 3 being rather narrow, whereas the $\alpha$ histogram of Fig. 10 has a fairly extended range.

A 2D histogram can be built from Fig. 12 by defining 2D bins and counting the number of points falling inside each $2 \mathrm{D}$ bin. This histogram is represented in Fig. 13. Its envelope is the joint PDF of the two random variables $\alpha$ and $B$, that we denote as $p(\alpha, B)$. The shape of the envelope (and Fig. 12) shows that these two variables are strongly correlated. The magnetic field PDF (the envelope of the histogram of Fig. 11) is the marginal PDF

$p(B)=\int p(\alpha, B) \mathrm{d} \alpha$.

Similarly, the envelope of Fig. 10 is the other marginal PDF, $p(\alpha)$.

For the benefit of the reader, we give the definition of the joint PDF of two random variables in Appendix A, and the related marginal PDFs (see also Papoulis 1965, Chap. 6).

\subsection{Discussion of the magnetic field PDF}

Confusion is encountered in the literature about the definition of the magnetic field PDF. This comes from the quiet Sun magnetic field being a complex quantity, having a PDF for both its field strength and magnetic filling factor, at least in data interpretation where these two quantities are determined in each pixel, 


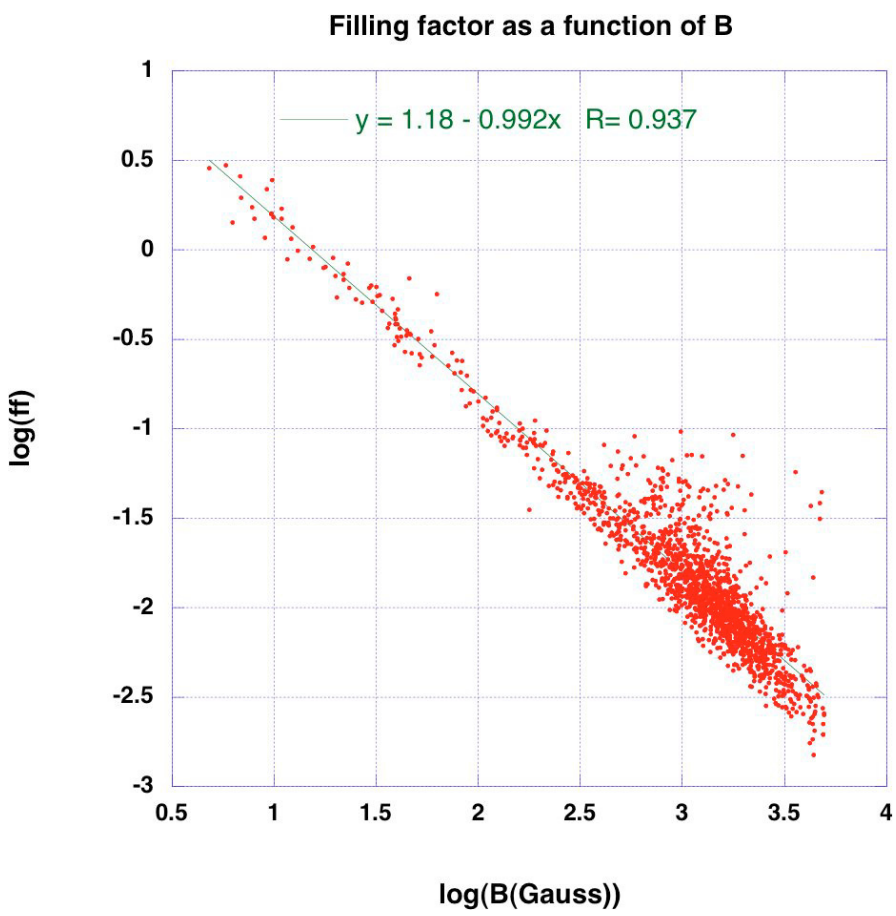

Fig. 12. Behavior of the magnetic filling factor $\alpha$ as a function of the magnetic field strength $B$. The linear fit can also be expressed as $\alpha=B_{1} / B$, with $B_{1}=15 \mathrm{G}$.

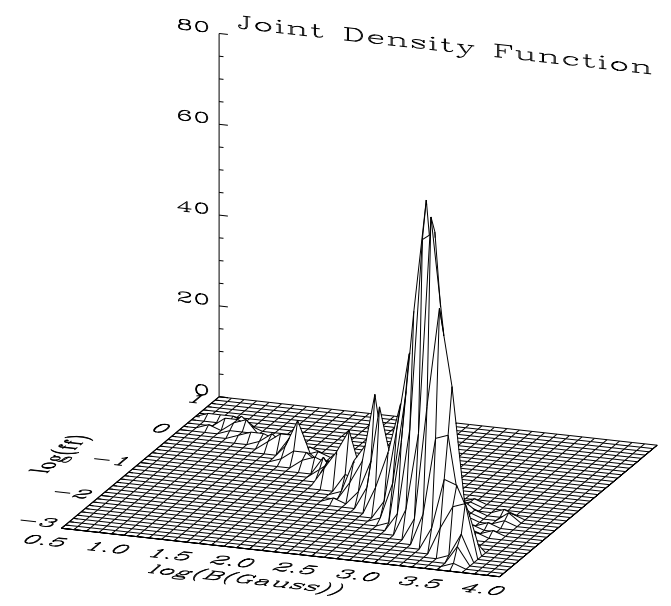

Fig. 13. 2D histogram of the magnetic filling factor and of the magnetic field strength $B$, each pair of them known in each solar pixel. This figure is a $3 \mathrm{D}$ representation of the number of points in Fig. 12. The joint PDF $p(\alpha, B)$ is the envelope of the 2D histogram. The two marginal density functions $p(\alpha)$ and $p(B)$ for each of the two random variables $\alpha$ and $B$ are the envelopes of the two histograms given in Figs. 10 and 11 respectively.

the magnetic filling factor $\alpha$ representing the fraction of the resolution element covered by the magnetic field $B$. From the modeling point of view, the necessity of introducing the magnetic filling factor $\alpha$ is less evident, and one has to carefully examine the modeling conditions, i.e. with or without filling factor, before comparing the magnetic field PDFs. Thus, the following discussion will be divided into several parts corresponding to different approaches.

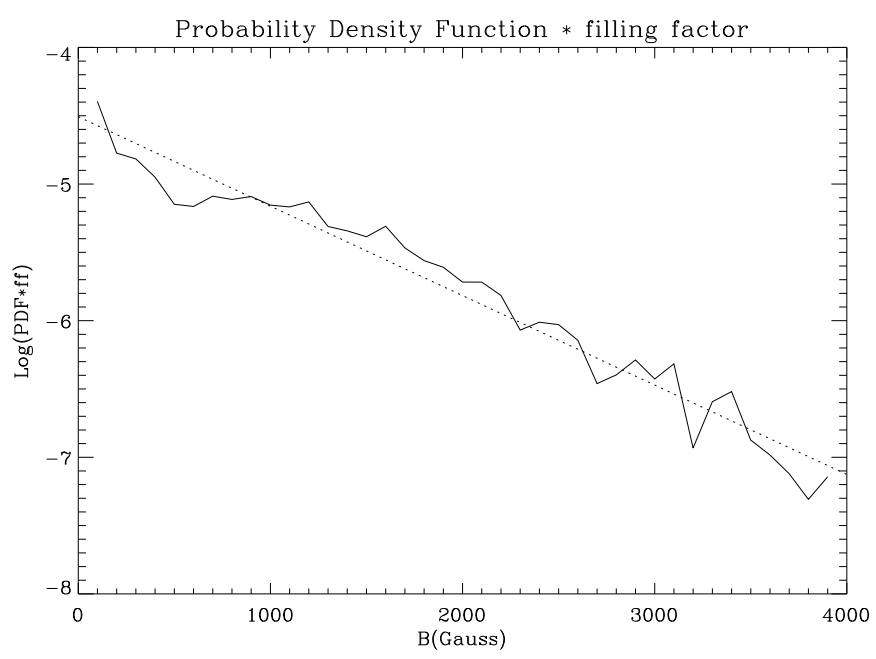

Fig. 14. Weighted histogram of the magnetic field strength. For each bin of the magnetic field strength histogram of Fig. 11, the count number has been multiplied by the average magnetic field filling factor for this magnetic field strength $\alpha=B_{1} / B$, with $B_{1}=15 \mathrm{G}$ following the linear fit in Fig. 12. The envelope of this histogram is then $\bar{\alpha}_{B} p(B)$, where $\bar{\alpha}_{B}$ is the average filling factor conditioned by $B$, and $p(B)$ is the magnetic field PDF of Fig. 11. The linear fit corresponds to an exponential decrease of $\bar{\alpha}_{B} p(B)$ as $\exp \left(-B / B_{0}\right)$, with $B_{0}=660 \mathrm{G}$.

\subsubsection{Comparison with Sánchez Almeida's definition}

For the interpretation of observations concerning the solar internetwork, Sánchez Almeida et al. (2003) introduce a quantity referred to as "magnetic field PDF" and defined as being "proportional to the sum of filling factors of all those measurements (i.e. of field strength $B$ ) in the bin $[B, B+\mathrm{d} B]$ ". In Sánchez Almeida (2007), this definition is rephrased as "the fraction of quiet Sun occupied by magnetic field of each strength". The same definition is used in Domínguez Cerdeña et al. (2006a,b). An explicit definition of this quantity is given in Eq. (14) of Martínez González et al. (2008a). Starting from this equation, we find that this so-called PDF can be written as $\bar{\alpha}_{B} p(B)$, with $p(B)$ the magnetic field marginal PDF introduced above and

$\bar{\alpha}_{B}=E_{B}(\alpha)=\int \alpha p(\alpha \mid B) \mathrm{d} \alpha$.

Here, $p(\alpha \mid B)$ is the PDF of $\alpha$, conditioned by the value of $B$. Hence, $\bar{\alpha}_{B}$ is the average magnetic filling factor, conditioned by the value of $B$. We recall that we have the relation

$p(\alpha, B)=p(\alpha \mid B) p(B)$,

Martínez González et al. (2008a) rightly mention that their PDF "takes into account the filling factor". We think that it is not a good idea to call the product $\bar{\alpha}_{B} p(B)$ a PDF. It certainly does not have the normalization property that one expects from a PDF.

We now explain why this quantity $\bar{\alpha}_{B} p(B)$ is very useful for solar polarization modeling, although it is not a true magnetic field PDF. For this purpose, let us recall that the first Stokes parameter $I$ is the specific intensity of radiation in erg $/ \mathrm{cm}^{2} / \mathrm{s} / \mathrm{sr} / \mathrm{Hz}$. This means that the energy $\mathrm{d} W$ emitted by the elementary surface $\mathrm{d} S$ during the elementary time interval $\mathrm{d} t$ in the elementary solid angle $\mathrm{d} \Omega$ and frequency interval $\mathrm{d} v$ is

$\mathrm{d} W=I \mathrm{~d} S \mathrm{~d} t \mathrm{~d} \Omega \mathrm{d} v$

The other Stokes parameters $Q, U, V$ have the same unit. For this reason, when computing, say, the average emitted $\bar{Q}$, starting 
from the known $Q(B)$ corresponding to the magnetic field $B$, we must take into account that only a fraction $\alpha$ of the elementary surface is occupied by the magnetic field, leading to

$\bar{Q}=\int \alpha Q(B) p(\alpha, B) \mathrm{d} \alpha \mathrm{d} B$.

For simplicity we assume that Stokes $Q$ only depends on the magnetic field strength. Using Eq. (12), we obtain

$\bar{Q}=\int Q(B) \bar{\alpha}_{B} p(B) \mathrm{d} B$

which demonstrates the exact physical meaning of the quantity $\bar{\alpha}_{B} p(B)$. But as discussed above this quantity is not the magnetic field PDF, a denomination that has to be reserved to $p(B)$. We suggest calling the product $\bar{\alpha}_{B} p(B)$ the "magnetic field contribution function" and denoting it as $C(B)$ (see also Appendix A).

\subsubsection{Analytical fit giving the magnetic field PDF}

In Fig. 14 we have plotted the histogram that has $\bar{\alpha}_{B} p(B)$ as envelope, for comparison with Fig. 6 (right) of Martínez González et al. (2008a) and with Fig. 3 of Sánchez Almeida et al. (2003) (although this last figure concerns longitudinal magnetic fields). It can be seen that the agreement with the IR data recommended by Martínez González et al. (2008a) is fairly good, although our field strengths are a bit higher. We agree with Martínez González et al. (2008a) on the linear behavior of the histogram (in log-lin coordinates). The linear fit of our data is

$\bar{\alpha}_{B} p(B)=A \mathrm{e}^{-\frac{B}{B_{0}}}$,

where $A$ is a constant to be determined by normalization, and $B_{0}=660 \mathrm{G}$. Assuming that $\bar{\alpha}_{B}=B_{1} / B$ (see Eq. (9)), imposing $\alpha \leq 1$, i.e. $B \geq B_{1}$, since $\alpha>1$ is unphysical, we obtain

$A=\frac{1}{B_{0}\left(1+B_{0} / B_{1}\right)} \mathrm{e}^{\frac{B_{1}}{B_{0}}}$,

so that finally

$p(B)=\frac{B}{B_{1} B_{0}\left(1+B_{0} / B_{1}\right)} \mathrm{e}^{\frac{B_{1}-B}{B_{0}}}$,

with $B_{1}=15 \mathrm{G}, B_{0}=660 \mathrm{G}$, and $B \geq B_{1}$. Figure 15 displays the histogram of Fig. 11 fitted with this function.

\subsubsection{Comparison with volume-filling PDFs}

Let us call "volume-filling PDF", the PDF of a magnetic field present everywhere in the medium. In this case $\alpha=1$ and the PDFs proposed in the literature have to be compared with the envelope of our local average magnetic field $\alpha B$ histograms. One also has to carefully examine whether the proposed PDF concerns the absolute field strength or the longitudinal field that is one component only of the field vector. We note that the consideration of the volume-filling longitudinal field is quite relevant, because this is the magnetic flux. We also note that in the case of a volume-filling magnetic field, the magnetic field PDF $p(B)$ is indeed the fraction of the solar surface occupied by fields with values between $B$ and $B+\mathrm{d} B$ (see the detailed discussion in Appendix A).

In this category, one finds the determination of the magnetic flux PDF by Stenflo \& Holzreuter (2003). As the flux distribution is derived from magnetograms, it has to be compared with

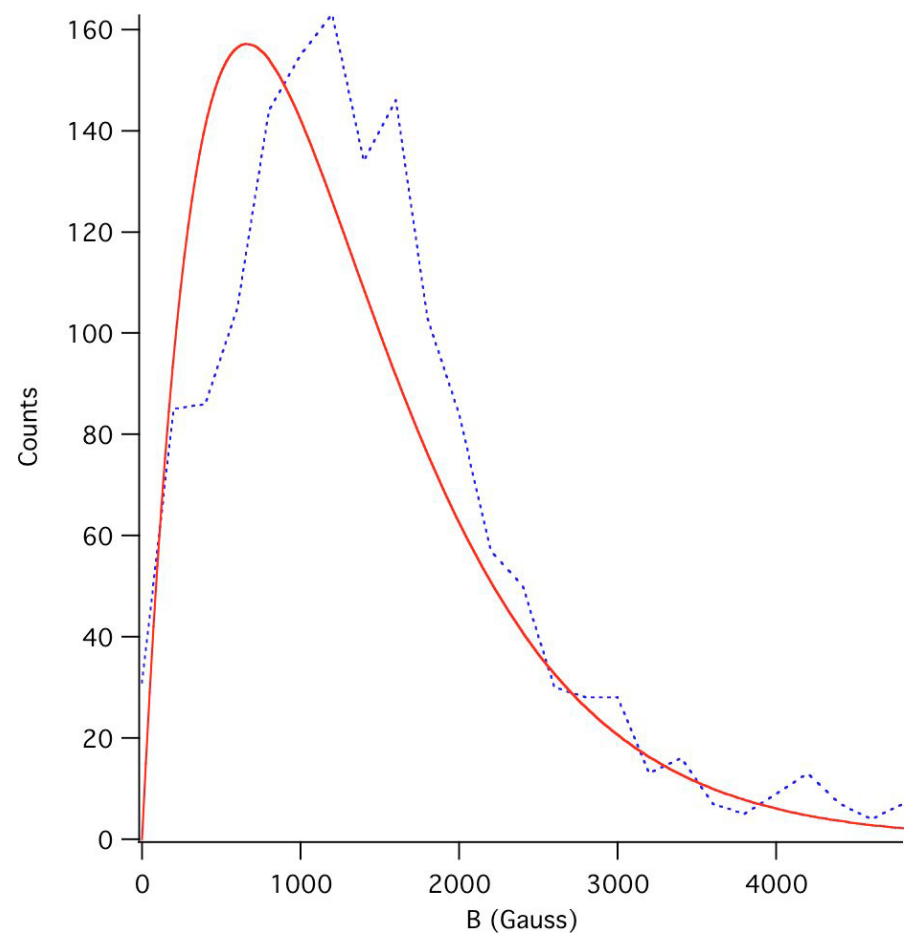

Fig. 15. Fit of magnetic field strength histogram of Fig. 11 by the magnetic field PDF $p(B)$ derived from the linear fits of Figs. 12 and 14. This magnetic field PDF is given by Eq. (18).

our Figs. 2 and 7. The widths of the distributions (13.5 G ours, $17.0 \mathrm{G}$ theirs) are comparable and in good agreement with the polarimetric accuracy of the corresponding measurements. The authors consider the wings of the distribution in detail. Probably, the wings are caused by network pixels that bear a stronger field and are visible in their magnetograms (Fig. 1). Such pixels cannot be avoided in the measurements. Our distribution also has broader wings than Gaussian, visible in logarithmic scale in Fig. 16, but we have a much lower pixel number in our analysis. In the same category one finds the theoretical study by Berrilli et al. (2008). This is also a longitudinal flux PDF to be compared with our Figs. 2 and 7. As is visible in Fig. 16, we do not have enough pixels to validate or invalidate this model with our observations.

We now turn to the field strength distribution. Inspired by the exponential PDF obtained by Martínez González et al. (2008a), Trujillo Bueno et al. (2004) introduced an exponential distribution for the volume-filling field strength, $p(B)=\exp \left(-B / B_{0}\right) / B_{0}$, to fit a series of Hanle effect measurements of the turbulent quiet Sun magnetic field. In addition, the field is assumed to be microturbulent with an isotropic angular distribution. With $B_{0}=130 \mathrm{G}$, the fit is rather satisfactory. It should also be mentioned that the same data can be fitted almost equally well with a single-valued magnetic field (PDF in the form of a Dirac distribution) with a strength of $B=60 \mathrm{G}$. Our $B_{0}$ value of $660 \mathrm{G}$ differs notably, however, from this $130 \mathrm{G}$ value, because they assume $\alpha=1$ (volume-filling magnetic field) in their simulation. This may also be due to the shape of our PDF that decreases towards zero at the axis origin, as well as our $\alpha B$ histogram in Figs. 3 and 8, as does the Maxwellian distribution function, whereas their theoretical distribution does not. Considering our result of $\alpha=B_{1} / B$ with $B_{1}=15 \mathrm{G}$, the filling factor remains close to unity for weak fields as those detected by Hanle effect interpretation, so that their hypothesis of volumefilling may be found coherent with our results for weak fields. 


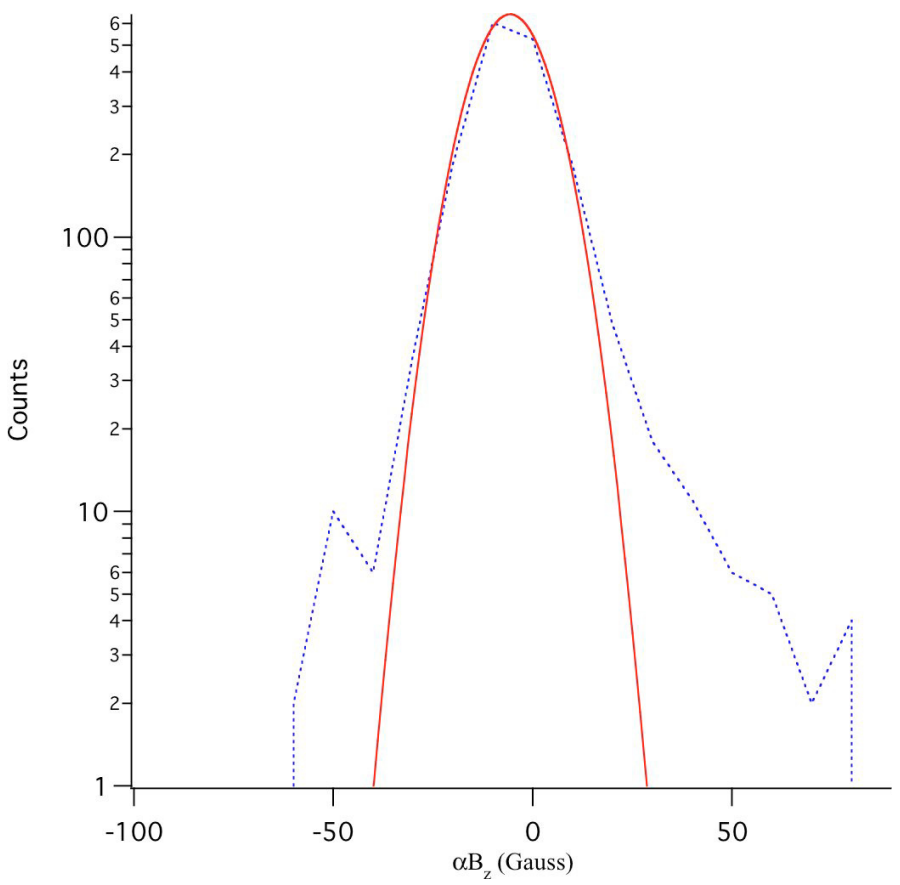

Fig. 16. Same as Fig. 7, but with the number of pixels per bin (counts) plotted on a logarithmic scale to highlight the distribution in the wings.

As mentioned in our conclusion, the derived PDF may depend on the sensitivity of the magnetic field measurement method (Hanle effect is sensitive to weak and unresolved fields, Zeeman effect is sensitive to the strongest fields). The mean value $B=60 \mathrm{G}$ that they derive under the hypothesis of volume-filling and singlevalued microturbulent field remains comparable to our mean value $\alpha B=15 \mathrm{G}$.

\subsubsection{Comparison with HINODE results}

We now discuss the results by Orozco Suárez et al. (2007b) and Ishikawa \& Tsuneta (2009). Both papers show a magnetic field strength PDF with a maximum in the $\mathrm{hG}$ range. A careful examination shows that their PDF definition is correct and is the same as ours, and that the differences in the results (our PDF peaks at higher field strengths) have to be assigned to a difference in the measurement of the magnetic filling factor. All these studies apply a Milne-Eddington inversion to the spectropolarimeteric data, with filling factor. As mentioned in Bommier et al. (2007), only the local average magnetic field strength $\alpha B$ can be retrieved from this inversion. It can be verified that both works agree when looking at this product $\alpha B$ : their field strengths are lower than ours, but their filling factor are higher than ours, so that the order of magnitude of their product is the same. We emphasize that their filling factor $\alpha$ is defined by the rate of stray light, which is also assumed to be the proportion of light coming from the unmagnetic region (note that their $\alpha$ corresponds to our $1-\alpha$ )

The question lies in the filling factor method of measurement. Ours is described above and is performed independently of the Milne-Eddington inversion. They derive the filling factor by comparing, in a first step, local intensity profiles (no polarization at that step) with average intensity profiles assumed to represent the zero field situation. This is the method introduced by Skumanich \& Lites (1987) and discussed by Lites \& Skumanich (1990). Depending of the activity level of the map under study, this average profile is evaluated either by a global average or by a local average on the less active part on the map. In the present case of quiet Sun studies, this profile is determined by a local average evaluated around the pixel of interest. Considering that this approach gives a different result from ours, we are led to raise the question to know whether such a method really determines the nonmagnetic profile. If this average profile is taken from the whole map (global average) or from a less active but wide part of it, we note that the local physical parameters at the pixel under study (temperature, density) may not be fully taken into account in the nonmagnetic global profile. And if this average is performed on the neighbor pixels only as in the abovementioned studies, we raise the question: is the local average really nonmagnetic with respect to the considered pixel?

\subsubsection{What can be expected from Fe I 6302.5 measurements?}

When the quiet Sun magnetic field strength $B$ is discussed, this magnetic field also has a magnetic filling factor $\alpha$. The question is how to determine $\alpha$ and $B$ separately. As discussed in Bommier et al. (2007), it is not possible to determine $\alpha$ and $B$ separately from inversion of Fe I $6302.5 \AA$ data, but only their product $\alpha B$. This is because, for solar magnetic field strengths and for a visible line like Fe I $6302.5 \AA$, the Zeeman splitting unit $\Delta \lambda_{\mathrm{B}}$ remains smaller or close to the Doppler width $\Delta \lambda_{\mathrm{D}}$. This behavior can be expected for any line in the visible range. On the contrary, when the Zeeman components are separated, determining $\alpha$ and $B$ separately, by inversion, becomes possible. This is partly true at infrared wavelengths (see the discussion at the beginning of Sect. 5). That is why Martínez González et al. (2008a) are able to get reliable results by inverting infrared lines, and we get results in agreement with theirs by applying our direct $\alpha$ determination complementing the inversion of the visible Fe I $6302.5 \AA$ line. Examining the work of Martínez González et al. (2008a), it can be seen that they are not confident in the Fe I $6302.5 \AA$ results in separate $\alpha$ and $B$, because they find that these results depend on the initialization of the inversion; however, it can be seen in their paper that the magnetic flux, which is the longitudinal counterpart of the product $\alpha B$, remains unchanged whatever the initialization be. This confirms that the inversion of visible Fe I $6302.5 \AA$ data accurately recovers the local average magnetic field strength $\alpha B$, though it is not able to recover $\alpha$ and $B$ separately.

\section{Conclusions}

We find that the magnetic field filling factor $\alpha$ is related to the magnetic field strength $B$ by the simple approximate law $\alpha=B_{1} / B$ with $B_{1}=15 \mathrm{G}$. Moreover, we find that the magnetic field PDF can be expressed by the analytical law of Eq. (18). This means that the medium is complex and various field strengths may be encountered: (i) the 1500 Gauss field filling $1 \%$ of space as seen in the tails of the magnetic field distributions by Khomenko et al. (2003), Martínez González et al. (2006), Orozco Suárez et al. (2007b), Martínez González et al. (2008a), as also detected by Domínguez Cerdeña et al. (2003a,b, 2006a,b); (ii) the 150 Gauss field filling $10 \%$ of space as seen from incomplete Paschen-Back effect interpretation by López Ariste et al. (2007) and Sánchez Almeida et al. (2008); (iii) 20-50 Gauss field filling the major part of space, as seen from the Hanle effect interpretation (see the measurement review by Trujillo Bueno et al. (2004) and Trujillo Bueno et al. (2006) 
for the Sr I 4607 line, see also Bommier et al. (2006) for a series of $\mathrm{MgH}$ lines).

Because the $\mathrm{kG}$ internetwork field with very small filling factors were previously detected (Grossmann-Doerth et al. 1996; Sánchez Almeida \& Lites 2000), recent magnetoconvection modeling (Bushby et al. 2008) shows that fields stronger than the equipartition value could result from localized concentrations due to convective intergranular downflows. However, it should be noted that our results have to be taken with care because our field determination is based on a 2-component inversion, whereas we arrive at the vision of a complex medium where all the field strengths coexist.

Actually, each magnetic field determination (Zeeman, hyperfine structure, Hanle) has its own sensitivity domain. Because it is a linear effect, the Zeeman effect detects the stronger fields better even if they do not fill the whole space. In contrast, the Hanle effect, being highly nonlinear, cannot detect the strong fields. To prove this point, let us assume a 2-component atmosphere with a 2000 Gauss field filling $2 \%$ of space: $98 \%$ of the scattered radiation will not be depolarized, while $2 \%$ will only be depolarized by the Hanle effect. As a $2000 \mathrm{G}$ field corresponds to the saturation regime of the Hanle effect, the corresponding linear polarization is $1 / 5$ of the zero field one. As a result, the global polarization remains almost insensitive to strong intermittent magnetic fields. In contrast, the Hanle effect is sensitive to weak field filling the major part of space. The incomplete Paschen-Back effect is sensitive to hG fields, but also is unable to detect $\mathrm{kG}$ fields. The result of our paper is that the strong, intermediate, and weak fields cohabit, with different filling factors obeying the simple approximate law $\alpha=B_{1} / B$ with $B_{1}=15 \mathrm{G}$.

In a later paper of this series, we will analyze the centerto-limb variation of the quiet Sun polarization with ZIMPOL on THEMIS observations, in order to thoroughly investigate the field direction distribution function. Further observations with a better polarimetric accuracy are, however, needed to confirm the results obtained in the present work.

Acknowledgements. The authors are grateful and indebted to D. Gisler and A. Feller for having provided the ZIMPOL data reduction package adapted to THEMIS, and to the whole ZIMPOL and THEMIS teams and to J.O. Stenflo for having given them the possibility of using ZIMPOL on THEMIS. They are also grateful to the anonymous referee for the kind and very helpful comments. Thanks also to J. Adams for the excellent english language corrections. The ZIMPOL campaign at THEMIS was financed by the SNF grant 200020-117821. A.A.R. acknowledges financial support by the Spanish Ministry of Education and Science through project AYA2007-63881.

\section{References}

Berrilli, F., Del Moro, D., \& Viticchiè, B. 2008, A\&A, 489, 763

Bommier, V., Landi Degl'Innocenti, E., Feautrier, N., \& Molodij, G. 2006, A\&A, 458,625

Bommier, V., Landi Degl'Innocenti, E., Landolfi, M., \& Molodij, G. 2007, A\&A, 464, 323

Bushby, P. J., Houghton, S. M., Proctor, M. R. E., \& Weiss, N. O. 2008, MNRAS, 387,698

Domínguez Cerdeña, I., Sánchez Almeida, J., \& Kneer, F. 2003a, A\&A, 407, 741

Domínguez Cerdeña, I., Kneer, F., \& Sánchez Almeida, J. 2003b, ApJ, 582, L55 Domínguez Cerdeña, I., Sánchez Almeida, J., \& Kneer, F. 2006a ApJ, 636, 496 Domínguez Cerdeña, I., Almeida, J. S., \& Kneer, F. 2006b, ApJ, 646, 1421 Faurobert-Scholl, M. 1992, A\&A, 258, 521

Gandorfer, A. M., \& Povel, H. P. 1997, A\&A, 328, 381

Gandorfer, A. M., Steiner, H. P. P. P., Aebersold, F., et al. 2004, A\&A, 422, 703 Grossmann-Doerth, U., Keller, C. U., \& Schuessler, M. 1996, A\&A, 315, 610

Ishikawa, R., \& Tsuneta, S. 2009, A\&A, 495, 607

Khomenko, E. V., \& Collados, M. 2007, ApJ, 659, 1726

Khomenko, E. V., Collados, M., Solanki, S. K., Lagg, A., \& Trujillo Bueno, J. 2003, A\&A, 408, 1115
Landolfi, M., Landi Degl'Innocenti, E., \& Arena, P. 1984, Sol. Phys., 93, 269 Landi Degl'Innocenti, E. 1976, A\&AS, 25, 379

Landi Degl'Innocenti, E., \& Landolfi, M. 2004, Polarization in Spectral Lines (Dordrecht: Kluwer Academ. Publ.)

Lites, B., Socas-Navarro, H., Kubo, M., et al. 2007, PASJ, 59, 571

Lites, B. W., \& Skumanich, A. 1990, ApJ, 348, 747

Lites, B. W., \& Socas-Navarro, H. 2004, ApJ, 613, 600

Lites, B. W., Kubo, M., Socas-Navarro, H., et al. 2008, ApJ, 672, 1237

López Ariste, A., Tomczyk, S., \& Casini, R. 2006, A\&A, 454, 663

López Ariste, A., Martínez González, M. J., \& Ramírez Vélez, J. C. 2007, A\&A, 464,351

Maltby, P., Avrett, E. H., Carlsson, M., et al. 1986, ApJ, 306, 284

Martínez González, M. J., Collados, M., \& Ruiz Cobo, B. 2006, A\&A, 456, 1159 Martínez González, M. J., Collados, M., Ruiz Cobo, B., \& Beck, C. 2008a, A\&A, 477, 953

Martínez González, M. J., Asensio Ramos, A., López Ariste, A., \& Manso Sainz, R. 2008b, A\&A, 479, 229

Orozco Suárez, D., Bellot Rubio, L., \& del Toro Iniesta, J. C. 2007a, ApJ, 662, 31

Orozco Suárez, D., Bellot Rubio, L., del Toro Iniesta, J. C., et al. 2007b, ApJ, 670, L61

Papoulis, A. 1965, in Probability, Random Variables and Stochastic Processes (New-York: Mc Graw-Hill)

Ramírez Vélez, J. C., López Ariste, A., \& Semel, M. 2008, A\&A, 487, 731

Sánchez Almeida, J. 2007, ApJ, 657, 1150

Sánchez Almeida, J., \& Lites, B. W. 2000, ApJ, 532, 1215

Sánchez Almeida, J., Domínguez Cerdeña, I., \& Kneer, F. 2003, ApJ, 597, L177

Sánchez Almeida, J., Viticchié, B., Landi Degl'Innocenti, E., \& Berrilli, F. 2008, ApJ, 675, 906

Skumanich, A., \& Lites, B. W. 1987, ApJ, 322, 473

Socas-Navarro, H., \& Sánchez Almeida, J. 2002, ApJ, 565, 1323

Socas-Navarro, H., Martínez Pillet, V., \& Lites, B. W. 2004, ApJ, 611, 1139

Stenflo, J. O. 1973, Sol. Phys., 32, 41

Stenflo, J. O. 1982, Sol. Phys., 80, 209

Stenflo, J. O., \& Holzreuter, R. 2003, Current Theoretical Models and Future High Resolution Solar Observations: Preparing for ATST, ASP Conf. Ser., 286, 169

Trujillo Bueno, J., Shchukina, N., \& Asensio Ramos, A. 2004, Nature, 430, 326 Trujillo Bueno, J., Asensio Ramos, A., \& Shchukina, N. 2006, ASP Conf. Ser., 358,269

Vernazza, J. E., Avrett, E. H., \& Loeser, R. 1981, ApJS, 45, 635

\section{Appendix A: Statistical definitions}

The question of the magnetic field PDF of the solar internetwork is still a hot subject of discussion, with different observations leading to somewhat different answers. The meaning of magnetic field PDF is not always clear, in particular when the magnetic field is not present everywhere in the medium. We show here how an unambiguous definition of the magnetic field PDF can be given in this latter case.

We assume that we have some area at our disposal on the solar surface divided into a number $N$ of pixels. In each pixel, a value for the magnetic field vector $\boldsymbol{B}$ and a value for a filling factor $\alpha$, with $0 \leq \alpha \leq 1$, were determined by some inversion method. In each pixel, this factor yields the fraction of the surface where the magnetic field is present. In a quiet internetwork region, the values of $\boldsymbol{B}$ and $\alpha$ are changing from pixel to pixel in a way that cannot be predicted with certainty. This suggests treating these two quantities as random variables. If the value of $N$ is sufficiently high, we can hope to deduce from the data set statistical properties of the magnetic field and of the filling factor. This type of analysis is the purpose of the present paper, based on spectro-polarimetric data obtained with ZIMPOL on THEMIS. In the following we would like to give an overall picture of the basic definitions. All the concepts that are introduced below are standard elements in probability theory. We recommend Papoulis (1965) for a rigorous, yet easily readable, introduction to probability theory.

For simplicity in notation, we consider amplitude $B$ of the magnetic field instead of vector $\boldsymbol{B}$. We also assume that the 
random variables $B$ and $\alpha$ have a continuous range of values with $B \geq 0$ and $0 \leq \alpha \leq 1$. Henceforth, random variables are denoted with boldface characters to distinguish them from the outcome values of a given event. The continuity assumption implies that $N$ is infinite. The case of finite $N$ is considered below. We introduce the joint PDF $p(\alpha, B)$ defined by

$p(\alpha, B) \mathrm{d} \alpha \mathrm{d} B=$

$$
P\{\alpha \leq \boldsymbol{\alpha} \leq \alpha+\mathrm{d} \alpha, B \leq \boldsymbol{B} \leq B+\mathrm{d} B\} .
$$

As is clear from the definition, $p(\alpha, B) \mathrm{d} \alpha \mathrm{d} B$ is the probability that $\alpha$ has a value in the interval $[\alpha, \alpha+\mathrm{d} \alpha]$ and $\boldsymbol{B}$ a value in the interval $[B, B+\mathrm{d} B]$. We recall that $p(\alpha, B)$ is the second derivative, assumed to exist, of the joint probability distribution function $F(\alpha, B)$, i.e.

$p(\alpha, B)=\frac{\partial^{2} F(\alpha, B)}{\partial \alpha \partial B}$,

with

$F(\alpha, B)=P\{\boldsymbol{\alpha} \leq \alpha, \boldsymbol{B} \leq B\}$.

The joint distribution function satisfies $F(\alpha, B) \geq 0$ and $F(1, \infty)=1$.

The function $p(\alpha, B)$ satisfies $p(\alpha, B) \geq 0$ and the normalization condition

$\int_{0}^{1} \int_{0}^{\infty} p(\alpha, B) \mathrm{d} \alpha \mathrm{d} B=1$.

Starting from $p(\alpha, B)$, we can define the two marginal PDFs

$p(B)=\int_{0}^{1} p(\alpha, B) \mathrm{d} \alpha$,

$p(\alpha)=\int_{0}^{\infty} p(\alpha, B) \mathrm{d} B$.

These functions are the PDFs of $B$ and $\alpha$. It is easy to verify that they are normalized to unity, namely

$$
\begin{aligned}
& \int_{0}^{\infty} p(B) \mathrm{d} B=1 \\
& \int_{0}^{1} p(\alpha) \mathrm{d} \alpha=1 .
\end{aligned}
$$

They can be used to define the mean values

$\bar{B}=\int_{0}^{\infty} B p(B) \mathrm{d} B$,

$\bar{\alpha}=\int_{0}^{1} \alpha p(\alpha) \mathrm{d} \alpha$.

Very useful are the conditional PDFs $p(\alpha \mid B)$ and $p(B \mid \alpha)$, which are the PDF of $\alpha$, knowing $B$, and the PDF of $B$, knowing $\alpha$. They are defined by

$p(\alpha \mid B)=\frac{p(\alpha, B)}{p(B)}$,
$p(B \mid \alpha)=\frac{p(\alpha, B)}{p(\alpha)}$.

With these conditional PDFs, one can define conditional averages,

$\bar{\alpha}_{B}=\int_{0}^{1} \alpha p(\alpha \mid B) \mathrm{d} \alpha$,
$\bar{B}_{\alpha}=\int_{0}^{\infty} B p(B \mid \alpha) \mathrm{d} B$.
It is easy to verify that

$$
\begin{aligned}
& \bar{\alpha}=\int_{0}^{\infty} \bar{\alpha}_{B} p(B) \mathrm{d} B, \\
& \bar{B}=\int_{0}^{1} \bar{B}_{\alpha} p(\alpha) \mathrm{d} \alpha .
\end{aligned}
$$

The conditional average $\bar{\alpha}_{B}$ yields the mean value of the filling factor for magnetic fields with a value between $B$ and $B+\mathrm{d} B$. The product $\bar{\alpha}_{B} p(B)$ can be interpreted as the fraction of the surface occupied by magnetic fields between $B$ and $B+\mathrm{d} B$.

The concepts of joint PDF and conditional PDF are easy to grasp with a graphic representation. We introduce a rectangular coordinate system $(\alpha, B, z)$, with the axes $\alpha$ and $B$ defining the horizontal plane and $z$ the vertical axis. The joint PDF defines a surface $z=p(\alpha, B)$. If we intersect this surface by the vertical plane $B=B_{1}$, we obtain the profile of the conditional PDF $p\left(\alpha \mid B_{1}\right)$, as a function of $\alpha$. Similarly, a cut by the vertical plane $\alpha=\alpha_{1}$ yields the profile of the conditional PDF $p\left(B \mid \alpha_{1}\right)$, as a function of $B$.

In some spectro-polarimetric analysis one considers that the magnetic field is present everywhere in the medium. In this case the random variable $\alpha$ takes only the value $\alpha=1$. All the expressions given above hold with $p(\alpha, B)=\delta(\alpha-1) p(B)$, $\delta$ being the Dirac distribution. The conditional PDFs become $p(\alpha \mid B)=\delta(\alpha-1)$ and $p(B \mid \alpha)=p(B)$. For the Hanle effect, it is always assumed that $\alpha=1$ and often that the magnetic strength has a single value $B=B_{0}$. The corresponding joint PDF reduces to $p(\alpha, B)=\delta(\alpha-1) \delta\left(B-B_{0}\right)$.

For simplicity in the notation, we have retained the strength $B$ of the magnetic field as random variable. It is clear that all the definitions remain the same if we replace $B$ by the magnetic field vector $\boldsymbol{B}$. The vector $\boldsymbol{B}$ corresponds actually to three random variables, one for the strength and two for the direction. The differential element $\mathrm{d} B$ should be replaced $\mathrm{d}^{3} \boldsymbol{B}=B^{2} \sin \theta \mathrm{d} B \mathrm{~d} \theta \mathrm{d} \chi / 4 \pi$, with $\theta$ and $\chi$ the polar angles of the magnetic field direction in an appropriate reference frame.

An interesting quantity for the understanding of the Sun global energy budget is the mean magnetic energy density in the quiet internetwork. If the magnetic field is present everywhere, this mean energy is $\left\langle B^{2}\right\rangle / 8 \pi$ with

$$
\left\langle B^{2}\right\rangle=\int_{0}^{\infty} B^{2} p(B) \mathrm{d} B .
$$

If the field is not volume-filling, one should use

$$
\left\langle B^{2}\right\rangle=\int_{0}^{1} \int_{0}^{\infty} \alpha B^{2} p(\alpha, B) \mathrm{d} B \mathrm{~d} \alpha .
$$

This expression can be written

$$
\left\langle B^{2}\right\rangle=\int_{0}^{\infty} C(B) B^{2} \mathrm{~d} B
$$

with

$$
\begin{aligned}
C(B) & =\int_{0}^{1} \alpha p(\alpha, B) \mathrm{d} \alpha \\
& =\int_{0}^{1} \alpha p(\alpha \mid B) p(B) \mathrm{d} \alpha \\
& =\bar{\alpha}_{B} p(B) .
\end{aligned}
$$

Here, $\bar{\alpha}_{B}$ is the conditional mean value of $\boldsymbol{\alpha}$ defined in Eq. (A.13). The function $C(B)$ also appears in the calculation 
of the mean Stokes parameters $Q, U$, and $V$, when the magnetic field is not volume-filling (see Sect. 5). If the random variable $\alpha$ only takes the value $1, C(B)$ is simply the PDF of the random variable $\boldsymbol{B}$. If not, as suggested by Eq. (A.19), $C(B)$ could be referred to as the "magnetic field contribution function". The name "magnetic field PDF" should be reserved for the marginal PDF $p(B)$. One reason for not calling $C(B)$ a "magnetic field PDF" is that it is not normalized to unity. It is easy to check that the integral of $C(B)$ over $B$ is equal to the mean value $\bar{\alpha}$.

In the analysis of real data, the number $N$ of pixels is not infinite. Also the magnetic field and filling factor are determined with some error bar. What is usually inferred from the data are histograms of the "count number", i.e. of the number of pixels $n\left(B_{i}\right)$ in which the magnetic field strength takes a value between $B_{i}$ and $B_{i+1}=B_{i}+\Delta B$. Similarly histograms are being constructed with the number of pixels $n\left(\alpha_{j}\right)$ in which the filling factor takes a value between $\alpha_{j}$ and $\alpha_{j+1}=\alpha_{j}+\Delta \alpha$. The envelopes of these histograms, divided by $N \Delta B$ and $N \Delta \alpha$, yield the marginal PDFs $p(B)$ and $p(\alpha)$, in the limit $N \rightarrow \infty, \Delta B \rightarrow 0$, $\Delta \alpha \rightarrow 0$ with $N \Delta B$ and $N \Delta \alpha$ constants. One can also construct 2D-histograms corresponding to the joint PDF $p(\alpha, B)$. In each cell $\left[B_{i}, B_{i+1}\right] \times\left[\alpha_{j}, \alpha_{j+1}\right]$, one plots on the vertical axis, the number of pixels in which $B$ has a value in the interval $\left[B_{i}, B_{i+1}\right]$ and $\alpha$ has a value in the interval $\left[\alpha_{j}, \alpha_{j+1}\right]$. As in the continuous case, the conditional histograms are obtained by cutting the 2D-histogram with vertical planes. Examples of 1D and 2D histograms can be found in the present paper.
The mean magnetic energy takes the form

$$
\left\langle B^{2}\right\rangle=\sum_{i} C\left(B_{i}\right) B_{i}^{2} \Delta B
$$

with

$$
\begin{aligned}
C\left(B_{i}\right) & =\frac{1}{N \Delta B} \sum_{j} \alpha_{j} n\left(\alpha_{j}, B_{i}\right) \\
& =\left[\sum_{j} \alpha_{j} \frac{n\left(\alpha_{j}, B_{i}\right)}{n\left(B_{i}\right) \Delta \alpha} \Delta \alpha\right]\left[\frac{n\left(B_{i}\right)}{N \Delta B}\right],
\end{aligned}
$$

where $n\left(\alpha_{j}, B_{i}\right)$ is the number of pixels in which $\alpha_{j} \leq \alpha<\alpha_{j+1}$ and $B_{i} \leq B<B_{i+1}$. The definition of $C\left(B_{i}\right)$ in Eq. (A.23) is identical to the definition of the "magnetic field PDF" given in Eq. (14) in Martínez González et al. (2008a). We note here that $n\left(\alpha_{j}, B_{i}\right) /\left(n\left(B_{i}\right) \Delta \alpha\right)$ is the discrete version of the conditional PDF $p(\alpha \mid B)$, whereas $n\left(B_{i}\right) /(N \Delta B)$, is the discrete version of the marginal PDF $p(B)$. The summation over $j$ in the first square bracket of Eq. (A.23) yields the conditional mean value of $\alpha$ with $B$ in the interval $\left[B_{i}, B_{i+1}\right]$. When $\alpha$ takes only the value $\alpha=1, n\left(B_{i}\right) / N \Delta B$ is the fraction of the surface occupied by magnetic fields in the bin $\left[B_{i}, B_{i}+\Delta B\right]$, and this ratio is the discrete version of the magnetic field PDF. When the magnetic field is not present everywhere in the medium, the fraction of the surface occupied by magnetic fields with a value of $B$ in the bin $\left[B_{i}, B_{i}+\Delta B\right]$ is given by $C\left(B_{i}\right)$. As already stressed above, this function is not a PDF. 